\title{
A Framework for Multi-Stakeholder Decision-Making and Conflict Resolution
}

\author{
Alexander W. Dowling ", Gerardo Ruiz-Mercado ${ }^{\dagger}$, and Victor M. Zavala \\ ฯ Department of Chemical and Biological Engineering \\ University of Wisconsin, 1415 Engineering Dr, Madison, WI 53706, USA \\ †Office of Research and Development, National Risk Management Research Laboratory \\ U.S. Environmental Protection Agency, 26 West Martin Luther King Drive, Cincinnati, Ohio 45268, USA
}

\begin{abstract}
We propose a decision-making framework to compute compromise solutions that balance conflicting priorities of multiple stakeholders on multiple objectives. In our setting, we shape the stakeholder dissatisfaction distribution by solving a conditional-value-at-risk (CVaR) minimization problem. The CVaR problem is parameterized by a probability level that shapes the tail of the dissatisfaction distribution. The proposed approach allows us to compute a family of compromise solutions and generalizes multi-stakeholder settings previously proposed in the literature that minimize average and worst-case dissatisfactions. We use the concept of the CVaR norm to give a geometric interpretation to this problem and use the properties of this norm to prove that the CVaR minimization problem yields Pareto optimal solutions for any choice of the probability level. We discuss a broad range of potential applications of the framework that involve complex decision-making processes. We demonstrate the developments using a biowaste facility location case study in which we seek to balance stakeholder priorities on transportation, safety, water quality, and capital costs.
\end{abstract}

Keywords: multi-stakeholder, multiobjective, optimization, Pareto optimality, compromise, conditional value-at-risk.

\section{Introduction}

The design and control of engineering systems is a complex decision-making process in which multiple conflicting objectives of social, economic, and environmental nature must be taken into account. Multiobjective optimization techniques have been applied to many domains in chemical engineering which include separations [37, 55], crystallization [58], chromatography [73, 45, 36], control system tuning [28], and chemical reactors [59, 66, 41, 56, 44, 7, 31]. In such settings engineers seek to understand the trade-offs in technology performance metrics such as purity, recovery, operating cost, and yield. There has also been interest in balancing economic and environmental metrics using life cycle assessment methods to integrate process and enterprise-wide 
functions [34, 14, 29, 1, 69, 24]. Applications include integrated biorefineries and supply chains $[2,3,6,11,19,21,22,27,38,46,51,57,65,68,67,69]$, industrial facility location problems [40], balancing fresh water consumption, greenhouse gas emissions and/or waste water pollutants [52, 18, 19], design of water management and treatment networks [53, 63, 72], ecology/pollutant management $[10,57,15,60]$ and molecular design [49].

From our perspective, there are two fundamental problems that must be addressed in multiobjective optimization: dimensionality and ambiguity. While there exist computationally efficient strategies to compute elements of the Pareto set (such as the normal boundary intersection method [12, 33, 41]) it is well-known that these strategies become computationally intractable in settings with many objectives. This poses a limitation in modern studies, which must trade-off many metrics. For instance, a recent study by the U.S. Environmental Protection Agency (EPA) identified over a hundred metrics that can be used for evaluating different system designs [54]. Limitations associated to dimensionality might explain why the vast majority of multiobjective optimization studies reported in the literature are limited to two or three conflicting objectives [20,41, 63, 68, 74, 36, 67]. Dimensionality reduction techniques are often used to address settings with many objectives $[9,11,13,25,64,75]$.

From an ambiguity standpoint, it is important to realize that stakeholders have biased opinions and perceptions that will influence their final decision (i.e., which Pareto point to select or how to prioritize objectives). Stakeholder will also tend to disagree with others [61, 42, 16, 26, 4, 17, 5, 70]. For example, from an environmental policy-making point of view, the process of designing a new regulation that fulfills the priorities of all the stakeholders affected is a daunting task. This topic is rarely discussed in multiobjective optimization studies. The ultimate goal in a multi-stakeholder decision-making setting is to make a final decision that reaches a form of consensus (i.e., a compromise solution). Ideally, such a decision should be Pareto efficient in the sense that it represents a true trade-off between conflicting objectives and stakeholder priorities. In other words, an inefficient decision is one in which it is possible to improve one or more objectives without affecting the others or benefit one or more stakeholders without affecting the others.

In the presence of many conflicting objectives one can obtain compromise solutions without computing the entire Pareto set. A popular strategy for doing this is the VIKOR method [48]. This method has been used in several applications such as water resource planning and building design [47, 32]. The method generates multiple decision alternatives with different attributes (i.e., values for conflicting objectives). For each alternative the distance to the utopia point is evaluated (the utopia point is the ideal unreachable point where all the objectives are independently minimized). The alternatives are then ranked based on their distance to the utopia point. The TOPSIS method is also a popular approach used to determine compromise solutions [8]. This approach finds a decision by ranking alternatives in a way that minimizes the distance to the utopia point and that maximizes the distance to the nadir point (the nadir point is the point of worst objective values).

Neither the VIKOR nor the TOPSIS method capture the preferences of multiple stakeholders. The distance-based consensus method provides a mechanism to do this [23]. The idea is to construct alternatives that different stakeholders rank according to their own priorities. A final ranking is then selected that minimizes a disagreement metric, which measures the distance between the final ranking and the rankings provided by the stakeholders. This method provides a framework to capture 
the priorities of multiple stakeholders, but does not provide a formalism to construct alternatives. Moreover, in this setting the stakeholders lack of a quantifiable metric to rank alternatives and this can lead to ambiguity.

An interesting multi-stakeholder decision-making approach was recently presented in [62]. Here, the authors assume that stakeholders are polled to provide priority rules to be followed. From these rules, a unique set of weights that satisfy such rules is computed and these weights are then used to obtain a compromise solution. A disadvantage of this approach is that it can yield situations in which no unique feasible set of weights can be obtained that satisfy all of the stakeholder rules. In addition, this approach does not quantify how dissatisfied are stakeholders with a given compromise decision.

A key limitation of the multi-stakeholder approaches available in the literature is that they lack socio-economic and statistical foundations. Consequently, it is difficult to quantify how satisfied are stakeholders with a given decision, and how representative are the stakeholder opinions of those of the entire population. This is relevant in the context of sustainability where a large number of stakeholders, potentially spread over in geographical regions (e.g., communities, local/regional governments, industries) need to be taken into account. In [70] we presented a framework for multistakeholder optimization based on the concept of conditional-value-at-risk (CVaR). In this setting, we sample the preferences of a population of stakeholders in terms of weights that prioritize competing objectives. We then construct dissatisfaction functions as deviations from the stakeholder ideal solutions and note that the dissatisfactions can be interpreted as samples from a stakeholder population. Finally, we shape the dissatisfaction distribution by solving a CVaR minimization problem parameterized in the probability level. The CVaR framework generalizes multi-stakeholder optimization approaches in which compromise decisions are obtained by minimizing average and worst-case dissatisfactions $[16,30]$. A key advantage of this approach is that it does not require the computation of a Pareto set and thus can be used to address problems with many objectives and stakeholders. Moreover, the compromise solutions obtained from CVaR have practical statistical interpretations. In this work, we formalize the CVaR framework by proving that every solution of the CVaR minimization problem is a Pareto optimal solution of the original multiobjective optimization problem. We prove this result by exploiting properties of the so-called CVaR norm, which provides a geometric interpretation to the CVaR metric used in stochastic programming. We discuss different decision-making settings under which the CVaR framework can be used. We also provide numerical examples and a biowaste facility location study to illustrate the applicability of the framework and to discuss interpretations of the resulting compromise solutions.

The paper is structured as follows. In Section 2 we provide basic definitions while in Section 3 we describe the CVaR framework and establish Pareto optimality results. In Section 4 we discuss potential applications and provide numerical examples. In Section 5 we present a biowaste facility location case study. The paper closes in Section 6 with conclusions and directions for future work. 

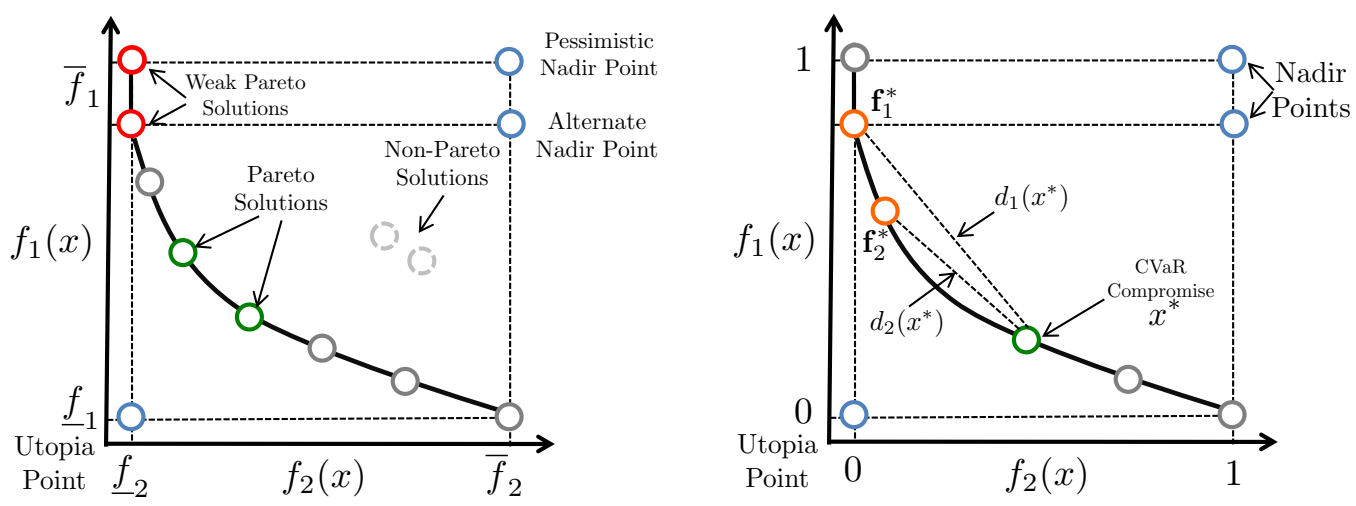

Figure 1: Sketch of multiobjective setting (left) and of multi-stakeholder setting (right).

\section{Basic Definitions}

We define the decision variable vector $x \in \Re^{n_{x}}$ and we assume this to lie in the feasible set $\mathcal{X} \subseteq \Re^{n_{x}}$ which we in turn assume to be compact and nonempty. We consider $n$ objective functions $f_{i}: \mathcal{X} \rightarrow \Re$ for $i \in \mathcal{O}=\{0 \ldots n-1\}$ and the corresponding objective vector $\mathbf{f}(x):=\left(f_{0}(x), f_{1}(x) \ldots, f_{n-1}(x)\right)$. We assume that the objective functions remain bounded in $\mathcal{X}$. We define the multiobjective optimization (MOO) problem,

$$
\min _{x \in \mathcal{X}}\left(f_{0}(x), f_{1}(x) \ldots, f_{n-1}(x)\right) .
$$

Throughout the paper we assume that the minimization operand implies global minimization. We also assume that the objective functions have been scaled so that their values lie in the interval $[0,1]$. This can be done by using the coordinates of the utopia and nadir points as follows. We define,

$$
\begin{aligned}
\underline{f}_{i} & :=\min _{x \in \mathcal{X}} f_{i}(x), \quad i \in \mathcal{O} \\
\underline{x}_{i} & :=\underset{x \in \mathcal{X}}{\operatorname{argmin}} f_{i}(x), \quad i \in \mathcal{O} .
\end{aligned}
$$

Here, the coordinates of the utopia point are given by $\underline{f}_{i}$ while those of the nadir point are given by:

$$
\bar{f}_{i}:=\max \left\{f_{i}\left(\underline{x}_{0}\right), f_{i}\left(\underline{x}_{1}\right), \ldots, f_{i}\left(\underline{x}_{n-1}\right)\right\}, \quad i \in \mathcal{O} \text {. }
$$

We scale the objectives as,

$$
f_{i}(x) \leftarrow \frac{f_{i}(x)-\underline{f}_{i}}{\bar{f}_{i}-\underline{f}_{i}}, i \in \mathcal{O} .
$$

We can prevent visiting regions beyond the nadir points (of no interest) by imposing the constraints:

$$
0 \leq f_{i}(x) \leq 1, i \in \mathcal{O}
$$

The multiobjective optimization setting is illustrated in Figure 1. We use the following standard definitions of weak Pareto optimality and Pareto optimality [43]: 
Definition 1 (Weak Pareto Optimality) A decision $x^{*}$ with objectives $f_{i}\left(x^{*}\right), i \in \mathcal{O}$ is a weak Pareto solution of MOO if there does not exist an alternate solution $\bar{x}$ with objectives $f_{i}(\bar{x}), i \in \mathcal{O}$ satisfying $f_{i}(\bar{x})<$ $f_{i}\left(x^{*}\right)$ for all $i \in \mathcal{O}$.

Definition 2 (Pareto Optimality) A decision $x^{*}$ with objectives $f_{i}\left(x^{*}\right), i \in \mathcal{O}$ is a Pareto solution of MOO if there does not exist an alternate solution $\bar{x}$ with objectives $f_{i}(\bar{x}), i \in \mathcal{O}$ satisfying $f_{i}(\bar{x}) \leq f_{i}\left(x^{*}\right)$ for all $i \in \mathcal{O}$ and at least one index $i$ satisfying $f_{i}(\bar{x})<f_{i}\left(x^{*}\right)$.

We have that any Pareto solution of MOO is also a weak Pareto solution. To see this we consider the following contradiction: assume $x^{*}$ is a Pareto solution, and thus no alternate decision $\bar{x}$ exists such that $f_{i}(\bar{x}) \leq f_{i}\left(x^{*}\right)$ for all $i \in \mathcal{O}$ and $f_{i}(\bar{x})<f_{i}\left(x^{*}\right)$ for at least one index $i$. If, in addition, we assume that $x^{*}$ is not weak Pareto, then there exists an alternate decision $\bar{x}$ such that $f_{i}(\bar{x})<f_{i}\left(x^{*}\right)$ for all $i \in \mathcal{O}$. This is a contradiction and thus $x^{*}$ must be a Pareto and a weak Pareto solution.

The set of all Pareto solutions for MOO is known as the Pareto set. There are different methods that can be used to compute elements of the Pareto set, such as the weighting method. The results associated to this method will become relevant in the following sections. Consider a weight vector $\mathbf{w} \in \Re^{n}$. We define the elements of $\mathbf{w}$ as $w_{i}$ and we assume that these satisfy the condition:

$$
\begin{aligned}
w_{i} & \geq 0, \quad i \in \mathcal{O} \\
\sum_{i \in \mathcal{O}} w_{i} & =1 .
\end{aligned}
$$

In some cases we will require the weights to satisfy the stronger condition:

$$
\begin{aligned}
w_{i} & >0, \quad i \in \mathcal{O} \\
\sum_{i \in \mathcal{O}} w_{i} & =1 .
\end{aligned}
$$

Consider now the weighted problem:

$$
\min _{x \in \mathcal{X}} \mathbf{w}^{T} \mathbf{f}(x)
$$

The solutions of this problem satisfy the following property.

Proposition 1 Let $x^{*}$ be a solution of the weighted problem (2.8). We have that:

i) If the weight vector $\mathbf{w}$ satisfies (2.6a) then $x^{*}$ is a weak Pareto solution of MOO.

ii) If the weight vector $\mathbf{w}$ satisfies (2.7a) then $x^{*}$ is a Pareto solution of MOO.

Proof: Because $x^{*}$ is optimal for (2.8) we have that $\mathbf{w}^{T} \mathbf{f}(x) \geq \mathbf{w}^{T} \mathbf{f}\left(x^{*}\right)$ for all $x \in \mathcal{X}$. For i) assume now that $x^{*}$ is not a weak Pareto solution. From Definition 1 we have that this implies that there exists an alternative $\bar{x} \in \mathcal{X}$ such that $f_{i}(\bar{x})<f_{i}\left(x^{*}\right)$ for all $i \in \mathcal{O}$. We thus have that $\mathbf{w}^{T} \mathbf{f}(\bar{x})<\mathbf{w}^{T} \mathbf{f}\left(x^{*}\right)$ for any weight vector $\mathbf{w}$ satisfying (2.6a). From the optimality of $x^{*}$ we have that the alternative $\bar{x}$ cannot exist and we thus have a contradiction. Consequently, $x^{*}$ must be a weak Pareto solution. To establish ii) assume now that $x^{*}$ is not a Pareto solution. From Definition 2 we have that this implies 
that there exists an alternative $\bar{x} \in \mathcal{X}$ such that $f_{i}(\bar{x}) \leq f_{i}\left(x^{*}\right)$ for all $i \in \mathcal{O}$ and $f_{i}(\bar{x})<f_{i}\left(x^{*}\right)$ for at least one $i \in \mathcal{O}$. We thus have that $\mathbf{w}^{T} \mathbf{f}(\bar{x})<\mathbf{w}^{T} \mathbf{f}\left(x^{*}\right)$ for any weight vector $\mathbf{w}$ satisfying (2.7a). From the optimality of $x^{*}$ we have that the alternative $\bar{x}$ cannot exist and we thus have a contradiction. Consequently, $x^{*}$ must be a Pareto solution.

We note that the nadir point is obtained by computing worst-case values resulting from the determination of the utopia point, as in (2.2) and (2.3). In other words, we obtain the nadir point coordinates by minimizing each individual objective and then obtaining the corresponding worstcase values for the objectives. This approach is equivalent to solving (2.1) with $w_{i}=1, w_{i^{\prime} \neq i}=0$ for $i, i^{\prime} \in \mathcal{O}$. From Proposition 1 , these single objective solutions are only weak Pareto solutions. Consequently, it is possible to achieve a further reduction in the other objectives (the ones corresponding to the zero weights) without compromising the original single objective. Because of this, the nadir point may be overly pessimistic and unnecessarily enlarges the decision space. This in turn impacts the scaling of the objectives in (2.4). In Sections 4.2.1 and 5 we illustrate this undesirable behavior numerically. To address this issue, we propose an alternate nadir point and prove that it is a Pareto solution. We will also demonstrate that this nadir point can significantly shrink the decision space and can thus aid decision-making.

Definition 3 (Alternate Nadir Point) Let $\underline{x}_{i}, \underline{f}_{i}$ be defined as in (2.2) for all $i \in \mathcal{O}$. For each $i \in \mathcal{O}$ let the altenate nadir solution $\underline{x}_{i}^{*}$ be defined as:

$$
\begin{array}{r}
\underline{x}_{i}^{*}:=\underset{x \in \mathcal{X}}{\operatorname{argmin}} \mathbf{w}^{T} \mathbf{f}(x) \\
\text { s.t. } f_{i}(x) \leq \underline{f}_{i},
\end{array}
$$

where $\mathbf{w}$ satisfies $w_{i}=0$ and $w_{i^{\prime} \neq i}>0$. The alternate nadir point $\overline{\mathbf{f}}^{*}$ with elements $\bar{f}_{i}^{*}$ is defined as:

$$
\bar{f}_{i}^{*}:=\max \left\{f_{i}\left(\underline{x}_{0}^{*}\right), f_{i}\left(\underline{x}_{1}^{*}\right), \ldots, f_{i}\left(\underline{x}_{n-1}^{*}\right)\right\}, \quad i \in \mathcal{O} \text {. }
$$

Proposition 2 The alternate nadir solutions $\underline{x}_{i}^{*}, i \in \mathcal{O}$ are Pareto solutions of MOO.

Proof: Consider a fixed $i \in \mathcal{O}$. If $\underline{x}_{i}^{*}$ is not a Pareto solution, then there exists an alternate solution $\underline{\hat{x}}_{i}$ satisfying $f_{k}\left(\underline{\hat{x}}_{i}\right) \leq f_{k}\left(\underline{x}_{i}^{*}\right)$ for all $k \in \mathcal{O}$ and $f_{k}\left(\underline{\hat{x}}_{i}\right)<f_{k}\left(\underline{x}_{i}^{*}\right)$ for at least one $k \in \mathcal{O}$. However, per Definition 3, $\underline{x}_{i}^{*}$ is calculated from (2.9) such that $f_{i}\left(\underline{\hat{x}}_{i}\right) \leq \underline{f}_{i}$ and where $\underline{f}_{i}$ is the minimum of $f_{i}(x)$ for all $x \in \mathcal{X}$. We thus have that $f_{i}(x) \geq f_{i}\left(\underline{x}_{i}^{*}\right)$ holds for all $x \in \mathcal{X}$ (including $\underline{\hat{x}}_{i}$ ). Next, consider the rest of the objectives with indices $k \neq i$. If $f_{k}\left(\underline{\hat{x}}_{i}\right)<f_{k}\left(\underline{x}_{i}^{*}\right)$ for at least one $k$, then we have that $\mathbf{w}^{T} \mathbf{f}\left(\underline{\hat{x}}_{i}\right)<\mathbf{w}^{T} \mathbf{f}\left(\underline{x}_{i}^{*}\right)$, which implies $\underline{x}_{i}^{*}$ is not a solution to (2.9) and there is a contradiction. Consequently, $\underline{x}_{i}^{*}$ must be Pareto optimal.

The computation of the alternate nadir point requires a selection of weights. Such a selection is not unique and thus different alternate nadir solutions can be obtained. We note, however, that all of these alternatives are guaranteed to be Pareto solutions. Moreover, any of the nadir alternatives computed from (2.9) would be less pessimistic than the standard nadir point commonly used in the 
literature [43] (computed from (2.2) and (2.3)). Note also that even small nonzero weights guarantee this property and thus the alternate nadir point can be designed to simply prevent extreme degeneracies and volatility of the objectives resulting from the standard use of (2.2) and (2.3).

It is clear that computing all the elements in the Pareto set is computationally intractable, particularly when many objectives are considered. It is well known, however, that one can compute Pareto optimal compromise solutions by finding points that are closest in distance to the utopia point. This is often done by solving the minimization problem:

$$
\min _{x \in \mathcal{X}}\|\mathbf{f}(x)\|_{p}
$$

where $\|\cdot\|_{p}$ is the $L_{p}$ norm:

$$
\|\mathbf{f}(x)\|_{p}=\left(\sum_{i \in \mathcal{O}} f_{i}(x)^{p}\right)^{1 / p}
$$

with $p \geq 1$. We note that the absolute value of the argument is omitted because $f_{i}(x) \geq 0$ holds by construction. Pareto optimality of the compromise solutions is established in the following result.

Proposition 3 A solution $x^{*}$ of the minimum distance problem (2.11) is a Pareto solution of MOO.

Proof: From optimality of $x^{*}$ we have that $\|\mathbf{f}(x)\|_{p} \geq\left\|\mathbf{f}\left(x^{*}\right)\right\|_{p}$ for all $x \in \mathcal{X}$. Consider now the following contradiction. Assume decision $x^{*}$ is not a Pareto solution, and thus there exists an alternate decision $\bar{x}$ such that $f_{i}(\bar{x}) \leq f_{i}\left(x^{*}\right)$ for all $i \in \mathcal{O}$ and $f_{i}(\bar{x})<f_{i}\left(x^{*}\right)$ for at least one index $i$. From the definition of the $L_{p}$ norm we have that $\|\mathbf{f}(\bar{x})\|_{p}<\left\|\mathbf{f}\left(x^{*}\right)\right\|_{p}$ for all $p \geq 1$. From optimality of $x^{*}$, however, we have that $\bar{x}$ cannot exist and thus we have a contradiction $\square$.

\section{Multi-Stakeholder Decision-Making and CVaR Framework}

Multiobjective decision-making is inherently ambiguous in the sense that a final decision needs to be selected from the Pareto set. Such a decision is often made based on individual insights, expert knowledge, and/or preferences of a decision maker, which are ambiguous. Ambiguity introduces difficulty in assigning meaning to a final decision. For instance, while the compromise decision obtained from the minimum distance problem (2.11) has a geometric interpretation, it is not clear how to interpret such a compromise from a decision-maker satisfaction standpoint. In particular, a decision-maker might not necessarily want a point that is close to the utopia point, because her/his preferences are biased towards a certain objective or a set of objectives. Moreover, different decisionmakers might have different preferences and biases, and thus might disagree in the final decision to be selected. We address these issues by considering a multi-stakeholder setting, as proposed in [30].

Consider a number of stakeholders $m$ and assume that each stakeholder $j \in \mathcal{S}:=\{0, . ., m-1\}$ prioritizes the multiple objectives of $\mathrm{MOO}$ according to the weight vector $\mathbf{w}_{j} \in \Re^{n}$. We define the 
elements of $\mathbf{w}_{j}$ as $w_{j, i}$ and we assume them to satisfy either (2.6) or (2.7). Each stakeholder $j \in \mathcal{S}$ seeks to solve its individual weighted optimization problem:

$$
x_{j}^{*}:=\underset{x \in \mathcal{X}}{\operatorname{argmin}} \mathbf{w}_{j}^{T} \mathbf{f}(x) .
$$

The solution of problem (3.13) yields the objective vector $\mathbf{f}_{j}^{*}:=\mathbf{f}\left(x_{j}^{*}\right)$ with elements $f_{j, i}^{*}$ and corresponding weighted cost $\mathbf{w}_{j}^{T} \mathbf{f}_{j}^{*}$. This weighted cost is ideal in the sense that it assumes that stakeholder $j$ does not compromise with the rest of the stakeholders. From Proposition 1 we have that $x_{j}^{*}$ is a weak Pareto solution of MOO if $\mathbf{w}_{j}$ satisfies (2.6) and is a Pareto solution if $\mathbf{w}_{j}$ satisfies (2.7).

To deal with conflicting priorities among multiple stakeholders we need to measure how satisfied/dissatisfied are stakeholders with a given decision. To do so, we define the dissatisfaction function of stakeholder $j$ at decision $x$ as:

$$
\begin{aligned}
d_{j}(x): & =\mathbf{w}_{j}^{T}\left(\mathbf{f}(x)-\mathbf{f}_{j}^{*}\right) \\
& =\mathbf{w}_{j}^{T} \mathbf{f}(x)-\mathbf{w}_{j}^{T} \mathbf{f}_{j}^{*}
\end{aligned}
$$

We define the vector of dissatisfactions $\mathbf{d}(x):=\left(d_{1}(x), d_{2}(x), \ldots, d_{m-1}(x)\right)$. From optimality of $x_{j}^{*}$ with respect to (3.13) and of the associated weighted cost $\mathbf{w}_{j}^{T} \mathbf{f}_{j}^{*}$ we have that $\mathbf{w}_{j}^{T} \mathbf{f}(x) \geq \mathbf{w}_{j}^{T} \mathbf{f}_{j}^{*}$ and thus $d_{j}(x) \geq 0$ for all $x \in \mathcal{X}$ and $j \in \mathcal{S}$. Moreover, because the values of the objective functions $f_{i}(x)$ lie between zero and one and the weights satisfy either (2.6) or (2.7), we have that $d_{j}(x) \in[0,1]$ for all $x \in \mathcal{X}$.

To clarify these concepts consider that two arbitrary decisions $\bar{x}, x$ yield $d_{j}(\bar{x})<d_{j}(x)$ for a given stakeholder $j$. This means that stakeholder $j$ will be more satisfied under decision $\bar{x}$ than under decision $x$. Because of a possible conflict in priorities, however, another stakeholder $j^{\prime}$ might be less satisfied under decision $\bar{x}$ than under decision $x$ (i.e., $d_{j^{\prime}}(\bar{x})>d_{j^{\prime}}(x)$ ). To compute a stakeholder compromise we thus need to solve the multi-stakeholder optimization (MSO) problem:

$$
\min _{x \in \mathcal{X}}\left(d_{0}(x), \ldots, d_{m-1}(x)\right)
$$

Similar to the original MOO context of Section 2, we can find a solution to MSO by minimizing a metric of the stakeholder dissatisfactions. For instance, we can find a compromise solution my minimizing the $L_{p}$ norm of the dissatisfaction vector $\mathbf{d}(x)$. In the discussion that follows we will also use the concept of the scaled $L_{p}$ norm:

Definition 4 (Scaled $L_{p}$ norm). Consider a fixed decision $x \in \mathcal{X}$ and the associated dissatisfaction vector $\mathbf{d}(x)$. The scaled $L_{p}$ norm (denoted as $\left.L_{p}^{m}\right)$ of $\mathbf{d}(x)$ is defined as,

$$
\|\mathbf{d}(x)\|_{p}^{m}:=\left(\frac{1}{m} \sum_{j \in \mathcal{S}} d_{j}(x)^{p}\right)^{\frac{1}{p}}, p \geq 1 .
$$


The scaled $L_{p}$ norm has the following extreme cases,

$$
\begin{aligned}
\|\mathbf{d}(x)\|_{1}^{m} & =\frac{1}{m}\|\mathbf{d}(x)\|_{1}=\frac{1}{m} \sum_{j \in \mathcal{S}} d_{j}(x) \\
\|\mathbf{d}(x)\|_{\infty}^{m} & =\|\mathbf{d}(x)\|_{\infty}=\max _{j \in \mathcal{S}} d_{j}(x) .
\end{aligned}
$$

We note again that the absolute value is omitted from the norm definition because the dissatisfaction functions are non-negative by construction.

By working in the dissatisfaction space we can attribute more meaning to a compromise decision. For instance, we can find a compromise that minimizes the average stakeholder dissatisfaction [16]:

$$
\min _{x \in \mathcal{X}} \frac{1}{m} \sum_{j \in \mathcal{S}} d_{j}(x)
$$

From the above observations we have that this problem is equivalent to

$$
\min _{x \in \mathcal{X}}\|\mathbf{d}(x)\|_{1}^{m}
$$

The solution of problems (3.18) or (3.19) can also be interpreted as that of finding the closest point (in the $L_{1}$ norm) to the ideal point given by the collection of the ideal stakeholder costs $\mathbf{w}_{j}^{T} \mathbf{f}_{j}^{*}$.

Mehrotra and co-workers recently proposed to find a stakeholder compromise by minimizing the worst-case dissatisfaction among the stakeholders [30]:

$$
\min _{x \in \mathcal{X}} \max _{j \in \mathcal{S}} d_{j}(x)
$$

This problem is equivalent to the scaled $L_{p}$ norm minimization problem

$$
\min _{x \in \mathcal{X}}\|\mathbf{d}(x)\|_{\infty}^{m}
$$

We recently proposed to use the conditional value-at-risk ( $\mathrm{CVaR})$ as a generalized metric to create a multi-stakeholder framework that has the average (3.18) and worst-case (3.20) frameworks as extremes [70]. In this work we will attribute a geometric interpretation to CVaR that will help us establish Pareto optimality. We use the following definition of CVaR:

Definition 5 (CVaR) Consider a decision $x \in \Re^{n}$ and the discrete random variable $d(x)$ with outcomes $d_{0}(x), d_{1}(x), \ldots, d_{m-1}(x)$ and probabilities $p_{j}=\frac{1}{m}, j \in \mathcal{S}$. The CVaR of the random variable $d(x)$ with parameter $\alpha \in[0,1]$ is defined as,

$$
C \operatorname{VaR} R_{\alpha}(d(x)):=\min _{\nu \in \Re} \frac{1}{m} \sum_{j \in \mathcal{S}}\left(\nu+\frac{1}{1-\alpha}\left[d_{j}(x)-\nu\right]_{+}\right),
$$

where $[v]_{+}:=\max \{v, 0\}$ for $v \in \Re$. 
In the CVaR framework we compute a stakeholder compromise by solving the CVaR minimization problem:

$$
\min _{x \in \mathcal{X}} \operatorname{CVaR}_{\alpha}(d(x)) \Longleftrightarrow \min _{(x, \nu) \in \mathcal{X} \times \Re} \nu+\frac{1}{(1-\alpha) m} \sum_{j \in \mathcal{S}}\left[d_{j}(x)-\nu\right]_{+}
$$

It is well-known that this problem can be reformulated as,

$$
\begin{array}{cl}
\min _{\left(x, \nu, \phi_{j}\right) \in \mathcal{X} \times \Re \times \Re_{+}} & \frac{1}{m} \sum_{j \in \mathcal{S}}\left(\frac{1}{1-\alpha} \phi_{j}+\nu\right) \\
\text { s.t. } & \phi_{j} \geq d_{j}(x)-\nu, j \in \mathcal{S} .
\end{array}
$$

The CVaR formulation is motivated by the observation that we can interpret stakeholder opinions as samples from a population. The CVaR approach can then be used to shape the distribution of stakeholder dissatisfactions. In particular, CVaR penalizes the large dissatisfactions in the $(1-\alpha)$ tail of the stakeholder distribution. In other words, computing the CVaR of the dissatisfactions is equivalent to ordering the instances of $d(x)$ in increasing order, taking the $(1-\alpha)$ largest elements, and averaging them. Consequently, one can show that the CVaR solution converges to the worst-case solution as $\alpha \rightarrow 1$ and to the average solution as $\alpha \rightarrow 0$ [50]. The CVaR metric can also prevent the extreme conservatism of the worst-case solution. For instance, we might want to avoid a situation in which a single stakeholder dictates a solution. $\mathrm{CVaR}$ also help us explore if a decision changes when we discard certain elements of the population. Another advantage of the CVaR function is that it is convex and can be handled by standard optimization algorithms.

The $L_{p}$ and scaled $L_{p}$ norm interpretations also provide a generalized framework to compute compromise solutions (i.e., for varying values of $p$ ). Compromise solutions obtained with such norms, however, do not have statistical interpretations. The CVaR setting enables statistical interpretations of stakeholder compromise solutions and with that we also inherit other desirable properties. For instance, $\mathrm{CVaR}$ has asymptotic convergence as we increase the size of the stakeholder population [35]. In addition, it is possible to perform inference analysis on CVaR solutions (compute confidence intervals) [39]. This can help us evaluate, for instance, if the stakeholder group is a representative sample of the population or to determine an appropriate sample size or polling procedures that reduce variance. We can also analyze the impact of different opinion statistics on the nature of the compromise solution.

In previous work we have observed empirically that the solution of the CVaR problem (3.25) yields Pareto optimal solutions for MOO (2.1) for any value of $\alpha$ but no formal proof was established [70]. We now proceed to prove that this is indeed the case. Crucial to our development is the observation that $\mathrm{CVaR}$ also has a geometric interpretation, which was noticed in [50]. In that work, the authors introduced the notion of the CVaR norm of a vector. To explain this concept we define $\hat{\mathbf{d}}(x)$ as the vector of dissatisfactions $\mathbf{d}(x)$ with entries ordered from smallest to largest: $\hat{d}_{0}(x) \leq \hat{d}_{1}(x) \leq \ldots \leq \hat{d}_{m-1}(x)$. We use the following definition of the CVaR norm, that has been adapted to our context: 
Definition 6 (Scaled CVaR norm). Consider a decision $x \in \Re^{n_{x}}$ and dissatisfaction vector $\mathbf{d}(x)=$ $\left(d_{0}(x), d_{1}(x), \ldots, d_{m-1}(x)\right)$ with corresponding reordered vector $\hat{\mathbf{d}}(x)$. Define also the scalars $\alpha_{j}:=\frac{j}{m}, j \in \mathcal{S}$. The scaled CVaR norm of vector $\mathbf{d}(x)$ with parameter $\alpha_{j}$ is defined as,

$$
\langle\langle\mathbf{d}(x)\rangle\rangle_{\alpha_{j}}^{m}:=\frac{1}{m-j} \sum_{k=j}^{m-1} \hat{d}_{k}(x) .
$$

As can be seen, the CVaR norm with scalar $\alpha_{j}$ is the average of the $m-j$ largest entries of $\mathbf{d}(x)$. For each $j \in \mathcal{S}$ with associated $\alpha_{j}=\frac{j}{m}$, we can define the set of $m-j$ largest entries of $\mathbf{d}(x)$ as,

$$
\mathcal{K}_{\alpha_{j}}(x):=\left\{k \in \mathcal{S} \mid d_{k}(x) \geq \hat{d}_{j}(x)\right\}
$$

and we note that $\mathcal{K}_{\alpha_{j}}(x) \subseteq \mathcal{S}$ for all $j \in \mathcal{S}$ and $\mathcal{K}_{\alpha_{j}}(x)=\mathcal{S}$ if $j=0$. We also note that $\left|\mathcal{K}_{\alpha_{j}}(x)\right|=m-j$. We can use this set to express the CVaR norm as:

$$
\langle\langle\mathbf{d}(x)\rangle\rangle_{\alpha_{j}}^{m}=\frac{1}{m-j} \sum_{k \in \mathcal{K}_{\alpha_{j}}(x)} d_{k}(x) .
$$

We consider the following CVaR norm minimization problem:

$$
\min _{x \in \mathcal{X}}\langle\langle\mathbf{d}(x)\rangle\rangle_{\alpha}^{m}
$$

The definition of the CVaR norm is combinatorial in nature (entries need to be reordered). Consequently, (3.25) is not particularly amenable for computation. The following result, however, establishes an equivalence between the CVaR norm and the CVaR problem (3.22) and summarizes several properties for the CVaR norm. These properties have been established in [50] and we will use them to establish Pareto optimality of the solutions of the CVaR problem.

Property 1 For fixed $x$ consider the discrete random variable $d(x)$ with outcomes $d_{0}(x), d_{1}(x), \ldots, d_{m-1}(x)$, probabilities $p_{j}=\frac{1}{m}, j \in \mathcal{S}$, and the corresponding vector $\mathbf{d}(x)$. We have that:

i) $\langle\langle\mathbf{d}(x)\rangle\rangle_{\alpha}^{m}=C V a R_{\alpha}(d(x))$ for $\alpha \in[0,1]$.

ii) $\langle\langle\mathbf{d}(\mathbf{x})\rangle\rangle_{0}^{m}=\|\mathbf{d}(x)\|_{1}^{m}$

iii) $\langle\langle\mathbf{d}(x)\rangle\rangle_{\alpha}^{m}=\|\mathbf{d}(x)\|_{\infty}^{m}$ for $\frac{m-1}{m} \leq \alpha \leq 1$.

iv) For $\alpha$ such that $\alpha_{j}<\alpha<\alpha_{j+1}, j=0, \ldots, m-2$, the CVaR norm $\langle\langle\mathbf{d}(x)\rangle\rangle_{\alpha}^{m}$ satisfies

$$
\langle\langle\mathbf{d}(x)\rangle\rangle_{\alpha}^{m}=\mu\langle\langle\mathbf{d}(x)\rangle\rangle_{\alpha_{j}}^{m}+(1-\mu)\langle\langle\mathbf{d}(x)\rangle\rangle_{\alpha_{j+1}}
$$

with $\mu:=\frac{\left(\alpha_{j+1}-\alpha\right)\left(1-\alpha_{j}\right)}{\left(\alpha_{j+1}-\alpha_{j}\right)(1-\alpha)}$

v) $\langle\langle\mathbf{d}(x)\rangle\rangle_{\alpha}^{m}$ is a nondecreasing function of $\alpha \in[0,1]$. 
We highlight that another disadvantage of the $L_{p}$ norm is its high nonlinearity (with exception of the obvious choices $p=1, p=2$, and $p=\infty$ ). In [50] is was observed that the CVaR norm provides a piecewise linear approximation of the scaled $L_{p}$ norm, which is beneficial from a computational stand-point.

We now proceed to prove that the solution of the CVaR minimization problem (3.22) is indeed Pareto optimal for any choice of $\alpha \in[0,1]$. We begin by establishing the following key technical result.

Lemma 1 Consider decisions $\bar{x}$ and $x^{*}$ with dissatisfaction vectors $\mathbf{d}(\bar{x})$ and $\mathbf{d}\left(x^{*}\right)$. We have that:

$$
d_{j}(\bar{x})<d_{j}\left(x^{*}\right), \quad j \in \mathcal{S} \Longrightarrow\left\langle\langle\mathbf{d}(\bar{x})\rangle_{\alpha}^{m}<\left\langle\left\langle\mathbf{d}\left(x^{*}\right)\right\rangle\right\rangle_{\alpha}^{m}, \quad \alpha \in[0,1] .\right.
$$

Proof: We start by proving that $d_{j}(\bar{x})<d_{j}\left(x^{*}\right)$ for all $j \in \mathcal{S}$ implies that $\hat{d}_{j}(\bar{x})<\hat{d}_{j}\left(x^{*}\right)$ for all $j \in \mathcal{S}$. We pick a $j \in \mathcal{S}$ and consider decision $\bar{x}$ with subset $\mathcal{K}_{\alpha_{j}}(\bar{x}) \subseteq \mathcal{S}$ and note that,

$$
\hat{d}_{j}(\bar{x})=\min _{k \in \mathcal{K}_{\alpha_{j}}(\bar{x})} d_{k}(\bar{x}) .
$$

Similarly, for decision $x^{*}$ and subset $\mathcal{K}_{\alpha_{j}}\left(x^{*}\right) \subseteq \mathcal{S}$ we have

$$
\hat{d}_{j}\left(x^{*}\right)=\min _{k \in \mathcal{K}_{\alpha_{j}}\left(x^{*}\right)} d_{k}\left(x^{*}\right) .
$$

Next, we observe that $d_{j}(\bar{x})<d_{j}\left(x^{*}\right)$ for all $j \in \mathcal{S}$ implies that

$$
\min _{k \in \mathcal{K}} d_{k}(\bar{x})<\min _{k \in \mathcal{K}} d_{k}\left(x^{*}\right)
$$

for any subset $\mathcal{K} \subseteq \mathcal{S}$. Furthermore, from the definition of $\mathcal{K}_{\alpha_{j}}\left(x^{*}\right)$, we have that

$$
\min _{k \in \mathcal{K}} d_{k}\left(x^{*}\right) \leq \min _{k \in \mathcal{K}_{\alpha_{j}}\left(x^{*}\right)} d_{k}\left(x^{*}\right)
$$

for any subset $\mathcal{K} \subseteq \mathcal{S}$ of cardinality $|\mathcal{K}|=m-j$. We can thus establish that:

$$
\hat{d}_{j}(\bar{x}) \stackrel{(3.26)}{=} \min _{k \in \mathcal{K}_{\alpha_{j}}(\bar{x})} d_{k}(\bar{x}) \stackrel{(3.28)}{<} \min _{k \in \mathcal{K}_{\alpha_{j}}(\bar{x})} d_{k}\left(x^{*}\right) \stackrel{(3.29)}{\leq} \min _{k \in \mathcal{K}_{\alpha_{j}}\left(x^{*}\right)} d_{k}\left(x^{*}\right) \stackrel{(3.27)}{=} \hat{d}_{j}\left(x^{*}\right) .
$$

We thus have $\hat{d}_{j}(\bar{x})<\hat{d}_{j}\left(x^{*}\right)$ for all $j \in \mathcal{S}$. Consider now discrete values of $\alpha_{j}=\frac{j}{m}$ where $j \in \mathcal{S}$. At these points, $\langle\langle\mathbf{d}(x)\rangle\rangle_{\alpha_{j}}^{m}$ is computed by averaging $d_{k}(x), k \in \mathcal{K}_{\alpha_{j}}(x)$. Because we have established that $\hat{d}_{j}(\bar{x})<\hat{d}_{j}\left(x^{*}\right)$ holds, we have that $\langle\langle\mathbf{d}(\bar{x})\rangle\rangle_{\alpha_{j}}^{m}<\left\langle\left\langle\mathbf{d}\left(x^{*}\right)\right\rangle\right\rangle_{\alpha_{j}}^{m}$. From Property 1 we have that, for arbitrary $x$ and any $\alpha$ such that $\alpha_{j}<\alpha<\alpha_{j+1}, j=0, \ldots, m-2$, the CVaR norm $\langle\langle\mathbf{d}(x)\rangle\rangle_{\alpha_{j}}^{m}$ is computed by a linear combination of $\langle\langle\mathbf{d}(x)\rangle\rangle_{\alpha_{j}}^{m}$ and $\langle\langle\mathbf{d}(x)\rangle\rangle_{\alpha_{j}+1}^{m}$. We thus have that $\langle\langle\mathbf{d}(\bar{x})\rangle\rangle_{\alpha}^{m}\left\langle\left\langle\left\langle\mathbf{d}\left(x^{*}\right)\right\rangle\right\rangle_{\alpha}^{m}\right.$ for all $\alpha \in[0,1]$.

We can now establish our main result.

Theorem 1 Let $x^{*}$ be a solution of the CVaR problem (3.22). We have that:

i) If $\mathbf{w}_{j}, j \in \mathcal{S}$ satisfy (2.6) then $x^{*}$ is a weak Pareto solution of MOO (2.1) for any $\alpha \in[0,1]$.

ii) If $\mathbf{w}_{j}, j \in \mathcal{S}$ satisfy (2.7) then $x^{*}$ is a Pareto solution of MOO (2.1) for any $\alpha \in[0,1]$. 
Proof: Because $x^{*}$ is optimal for (3.25) we have that $\langle\langle\mathbf{d}(x)\rangle\rangle_{\alpha}^{m} \geq\left\langle\left\langle\mathbf{d}\left(x^{*}\right)\right\rangle\right\rangle_{\alpha}^{m}$ for any $x \in \mathcal{X}$. To establish i) assume $x^{*}$ is not a weak Pareto solution. This implies that there exists an alternate decision $\bar{x} \in \mathcal{X}$ such that $f_{i}(\bar{x})<f_{i}\left(x^{*}\right)$ for all $i \in \mathcal{O}$. We thus have that $\mathbf{w}_{j}^{T} \mathbf{f}(\bar{x})<\mathbf{w}_{j}^{T} \mathbf{f}\left(x^{*}\right)$ for any weight vector $\mathbf{w}_{j}$ satisfying (2.6) and $d_{j}(\bar{x})<d_{j}\left(x^{*}\right)$ for all $j \in \mathcal{S}$. From Lemma 1 we also have that $\langle\langle\mathbf{d}(\bar{x})\rangle\rangle_{\alpha}^{m}<\left\langle\left\langle\mathbf{d}\left(x^{*}\right)\right\rangle\right\rangle_{\alpha}^{m}$. We thus have that the alternate solution $\bar{x}$ cannot exist, which is a contradiction. Consequently, $x^{*}$ is weak Pareto optimal. To establish ii) assume $x^{*}$ is not a Pareto solution. This implies that there exists an alternate decision $\bar{x} \in \mathcal{X}$ such that $f_{i}(\bar{x}) \leq f_{i}\left(x^{*}\right)$ for all $i \in \mathcal{O}$ and $f_{i}(\bar{x})<f_{i}\left(x^{*}\right)$ for at least one $i$. We thus have that $\mathbf{w}_{j}^{T} \mathbf{f}(\bar{x})<\mathbf{w}_{j}^{T} \mathbf{f}\left(x^{*}\right)$ for any weight vector $\mathbf{w}_{j}$ satisfying (2.7) and $d_{j}(\bar{x})<d_{j}\left(x^{*}\right)$ for all $j \in \mathcal{S}$. From Lemma 1 we also have that $\langle\langle\mathbf{d}(\bar{x})\rangle\rangle_{\alpha}^{m}<\left\langle\left\langle\mathbf{d}\left(x^{*}\right)\right\rangle\right\rangle_{\alpha}^{m}$. We thus have that the alternate solution $\bar{x}$ cannot exist, which is a contradiction. Consequently, $x^{*}$ is Pareto optimal.

Corollary 1 Any solution $x^{*}$ of the CVaR problem (3.22) is a Pareto solution of MSO (3.15).

Proof: Follows directly from Lemma 1 and the arguments for the proof of Proposition 3.

We make the following remarks:

- The Pareto optimality result of Theorem 1 is of significance because it guarantees that any solution of the CVaR minimization problem is efficient in the sense that it truly represents a trade-off in the objectives for the original MOO (2.1). The result also points out that only weak Pareto optimality can be guaranteed if any of the stakeholders sets a weight of zero for one of the objectives. Of course, weak Pareto optimality is better than no Pareto optimality at all. The presence of zero weights represents the situation in which a stakeholder takes a extreme position on its preferences. We note that in some special cases it is possible to obtain a Pareto solution even if some of the weights are zero. For instance, consider the special case in which we minimize the average of dissatisfactions (i.e., $\alpha=\alpha_{0}=0$ and $\mathcal{K}_{\alpha_{0}}(x)=\mathcal{S}$ for any $x$ ). Then, as long as the weights satisfy the condition

$$
\frac{1}{m} \sum_{k \in \mathcal{S}} w_{k, i}>0, \quad i \in \mathcal{O},
$$

we will recover Pareto optimality. In other words, the average of the stakeholder weights is positive for each objective. Pareto optimality follows because such a condition implies that

$$
\frac{1}{m} \sum_{k \in \mathcal{S}} d_{k}(\bar{x})<\frac{1}{m} \sum_{k \in \mathcal{S}} d_{k}\left(x^{*}\right),
$$

which is the condition needed to establish a contradiction in Theorem 1 . In other words, even if a stakeholder takes a extreme position on one objective (i.e., assigns a zero weight), Pareto optimality will be guaranteed if another stakeholder does not assign a zero weight on the same objective. 
- For general values of $\alpha \neq 0$ Pareto optimality can also occur in some cases. For instance, consider the situation in which we have a decision $x^{*}$ with set $\mathcal{K}_{\alpha_{j}}\left(x^{*}\right)$ and that for any alternative $\bar{x}$ we have that $\mathcal{K}_{\alpha_{j}}(\bar{x})=\mathcal{K}_{\alpha_{j}}\left(x^{*}\right)=\mathcal{K}$. Then, provided that $\frac{1}{m-j} \sum_{k \in \mathcal{K}} d_{k}(\bar{x})<\frac{1}{m-j} \sum_{k \in \mathcal{K}} d_{k}(x)$ holds, we have Pareto optimality. Such a situation can occur, for instance, if the weights satisfy $\sum_{k \in \mathcal{K}} w_{k, i}>0$ for all $i \in \mathcal{O}$ because the CVaR norm takes the average over the elements of $\mathcal{K}$.

- From a practical stand-point we also highlight that one can always prevent degeneracies and weak Pareto solutions by assigning a sufficiently small value to zero weights.

- Theorem 1 also indicates that Pareto optimality can be established for any value of the probability level $\alpha$, which implies that we can compute elements of the Pareto set by spanning the interval $\alpha \in[0,1]$. Spanning this interval enable us to consider stakeholder compromise solutions with different statistical properties. We also recall that the extremes of this interval correspond to average and worst-case compromise solutions, which indicate that CVaR provides a generalized framework.

- Corollary 1 indicates that any solution of the CVaR problem is a Pareto solution of the multistakeholder problem MSO (even if there are zero weights). This indicates that the solution of the CVaR problem also represents a true trade-off in the stakeholder dissatisfaction space. Consequently, it represents an actual compromise among stakeholders.

\section{Uses and Properties of CVaR Framework}

In this section we discuss different settings under which the CVaR framework can be used. We also discuss features of the stakeholder compromise solutions using numerical examples.

\subsection{Decision-Making Settings and Applications}

The CVaR framework can be used as a general framework for conflict resolution in decision-making environments. In particular, it can provide useful information to stakeholders that facilitates discussions and accelerates consensus reaching. In particular, the CVaR framework:

- Provides a quantifiable metric that enables systematic comparisons of the effect of different compromise solutions on the dissatisfaction of the stakeholders. With this, it is possible to analyze if the decision is fair or if a decision strongly divides the stakeholder population (a subset is strongly satisfied and another set is strongly dissatisfied).

- Enables us to use opinions of specific communities to implicitly assign value to factors or metrics that cannot be easily monetized.

- Enables us to analyze how the opinion of individual stakeholders or if different stakeholder populations affect a final decision. This information is valuable for a policy maker that seeks to assess what would be the impact of informing a population on a given subject or try to target incentives to encourage certain stakeholders to change their position. 
- Enables us to find out if population opinions indeed affect a decision. For instance, there might be instances in which, because of engineering or physical constraints, the solution is not affected by stakeholder preferences and therefore it is unnecessary to engage in discussions.

- Enables us to understand if a certain stakeholder or subset of stakeholders fully dictates the nature of a decision and therefore the dissatisfaction of the rest of the stakeholders. In such a case, it would be sensible to reconsider a decision.

- Enables us to assess how polling procedures affect a decision. For instance, a given polling procedure can lead to solutions that are strongly conflicting compared to another, depending on how information is presented to the stakeholders. In some cases it is also possible that different polling procedures give the same decision.

From an application standpoint we provide the following examples. Consider decision-making settings in which:

- A set of engineers disagree on what economic, sustainability, and safety metrics should be considered in a design. The metrics are many so the computation of the Pareto set is impractical. The framework can be used to identify a compromise solution that captures different priorities for many objectives.

- A set of engineers disagree on the future prices of a commodity that should be used in a planning exercise and the prices are difficult/impossible to forecast. The framework can find a compromise solution when experts disagree on prices or other parameters, such as interest rates or depreciation methods, that are used to define objectives.

- A utility system of a chemical complex or a urban area seeks to distribute steam and chilled water to a set of consumers and faces a shortage of supply. The proposed framework can be used to shed demands in a way that minimizes collective dissatisfaction.

- A set of operators of a manufacturing facility that disagree on how to price equipment wear and safety, or commodities that are priced using immature or non-existing markets (e.g., water).

- A set of building occupants that disagree on what set-point should be used for their thermostats. The framework can be used to find set-points in a way that balance total energy use and individual comfort based on preferences of the stakeholders.

- A community has conflicting priorities on water quality, emissions, and health hazards related to an industrial facility that will be installed nearby. The framework can be used by either regulators or engineers to find compromise designs, operational policies, and/or regulations that account for both the community concerns and the facility's technical metrics.

- A government agency seeks to evaluate how the deployment of a given policy might affect conflicting interests of a community (e.g., land use, water use, safety). The framework can be used to assess the dissatisfaction of the community under different policy options. 
To provide some context on the scope of the proposed framework, we discuss how these capabilities can specifically facilitate activities at the U.S. EPA. On-going projects include brownfield cleanups $^{1}$, green infrastructure ${ }^{2}$, transportation improvements, community action for a renewed environment $(\mathrm{CARE})^{3}$, and sustainable tribal communities ${ }^{4}$, all of which involve complex decision-making processes.

The EPA provides grants to communities, states, tribes, and other stakeholders to identify solutions that prevent, assess, safely clean up, reuse, and improve green spaces, urban zones, and industrial lands. Any grant program must work with the involved stakeholders to develop and test sustainable solutions while assessing needs and outcomes, examining trade-offs, and tracking progress. The stakeholders use multiple social (life expectancy, water supply and sanitation, security), environmental (air and water quality, ecosystem diversity, residential and industrial energy use, exposures to pollutants), and economic (income and economic activity, employment, poverty, revenues) indicators for defining what a sustainable community means to them ${ }^{5}$. A multi-stakeholder framework can help mitigate ambiguity that results from all of these different perspectives. The framework can also help communities obtain tangible compromise solutions that resolve conflicts. In addition, the proposed framework can factor the opinions of multiple local and regional communities and quantify their effects on the final decision.

The framework can also provide interoperability features that integrate existing assessment tools, models, and indicator databases. For instance, when locating new facilities or investing in new infrastructure, stakeholders can use the framework to express their concerns on exposure and potential risks from environmental pollutants. Such a setting would integrate, for instance, the multistakeholder framework with the EPAs community-focused exposure and risk screening tool (CFERST) ${ }^{6}$. This would allow to effectively minimize exposure to pollutants with the highest risks, overlay demographic data for identifying susceptible populations, and observe potential impact of geographical location.

\subsection{Features and Interpretations of CVaR Compromise Solutions}

We now discuss some features and interpretations of compromise solutions through simple numerical examples. All the examples are available at http: / / zavalab.engr.wisc.edu/data.

\footnotetext{
${ }^{1}$ http: //www.epa.gov/brownfields

${ }^{2}$ http://www.epa.gov/green-infrastructure

${ }^{3}$ http: //www.epa.gov/communityhealth/community-action-renewed-environment-care-resources

${ }^{4}$ http: //www.epa.gov/tribal

${ }^{5}$ https://www.sustainablecommunities.gov/indicators

${ }^{6}$ http://www.epa.gov/healthresearch/community-focused-exposure-and-risk-screening-tool-c-ferst
} 


\subsubsection{Example 1}

Consider the two-objective problem:

$$
\begin{array}{ll}
\min _{x} & \left(x_{0}, x_{1}\right) \\
\text { s.t. } & x_{0}+2 x_{1} \geq 5 \\
& x_{0}, x_{1} \geq 0
\end{array}
$$

The ideal stakeholder solution for weights $\left(w_{0}, w_{1}\right)$ is given by:

$$
x^{*}:= \begin{cases}x_{0}=5, x_{1}=0 & \text { if } w_{0}<1 / 3 \\ x_{0}+2, x_{1}=5 & \text { if } w_{0}=1 / 3 \\ x_{0}=0, x_{1}=5 / 2 & \text { if } w_{0}>1 / 3\end{cases}
$$

The objectives correspond to the actual variables (i.e., $f_{0}(x)=x_{0}$ and $f_{1}(x)=x_{1}$ ). Using (2.2) and (2.3) we obtain the nadir standard point $\overline{\mathbf{f}}=[5,5]$. The alternate nadir point obtained using Definition 3 is $\overline{\mathbf{f}}^{*}=[5,5 / 2]$. The alternate point is clearly less pessimistic. The CVaR problem (3.22) can be formulated as:

$$
\begin{aligned}
\min _{x_{i}, \nu, \phi_{j}, f_{i}} & \frac{1}{m} \sum_{j \in \mathcal{S}}\left(\nu+\frac{1}{1-\alpha} \phi_{j}\right) \\
\text { s.t. } & x_{0}+2 x_{1} \geq 5 \\
& x_{0}, x_{1} \geq 0 \\
& f_{i}=x_{i}, \quad i \in \mathcal{O} \\
& \phi_{j} \geq d_{j}-\nu, \quad j \in \mathcal{S} \\
& \phi_{j} \geq 0, \quad j \in \mathcal{S} \\
& d_{j}=\sum_{i \in \mathcal{O}} w_{j, i}\left(\frac{f_{i}-f_{j, i}^{*}}{\bar{f}_{i}-\underline{f}_{i}}\right), \quad j \in \mathcal{S}
\end{aligned}
$$

where the expression for the dissatisfaction $d_{j}$ of stakeholder $j$ is obtained from the identity,

$$
\begin{aligned}
d_{j} & =\sum_{i \in \mathcal{O}} w_{j, i}\left(\frac{f_{i}-\underline{f}_{i}}{\bar{f}_{i}-\underline{f}_{i}}\right)-\sum_{i \in \mathcal{O}} w_{j, i}\left(\frac{f_{j, i}^{*}-\underline{f}_{i}}{\bar{f}_{i}-\underline{f}_{i}}\right) \\
& =\sum_{i \in \mathcal{O}} w_{j, i}\left(\frac{f_{i}-f_{j, i}^{*}}{\bar{f}_{i}-\underline{f}_{i}}\right) .
\end{aligned}
$$

Here, $f_{i, j}^{*}$ are the unscaled ideal stakeholder objectives. We assume we have two stakeholders with weights $\mathbf{w}_{\mathbf{0}}=[0.9,0.1]^{T}$ and $\mathbf{w}_{\mathbf{1}}=[0.1,0.9]^{T}$ and we solve (4.35) using the standard (pessimistic) and alternate nadir points. In Figure 2 we present the optimal solution and stakeholder dissatisfactions as a function of $\alpha$. We make the following observations:

- When $\alpha=0$ (minimize average dissatisfaction) we obtain that one stakeholder is fully satisfied while the other one is dissatisfied. In contrast, when $\alpha=1$ (minimize the maximum dissatisfaction), both stakeholders are equally satisfied. 

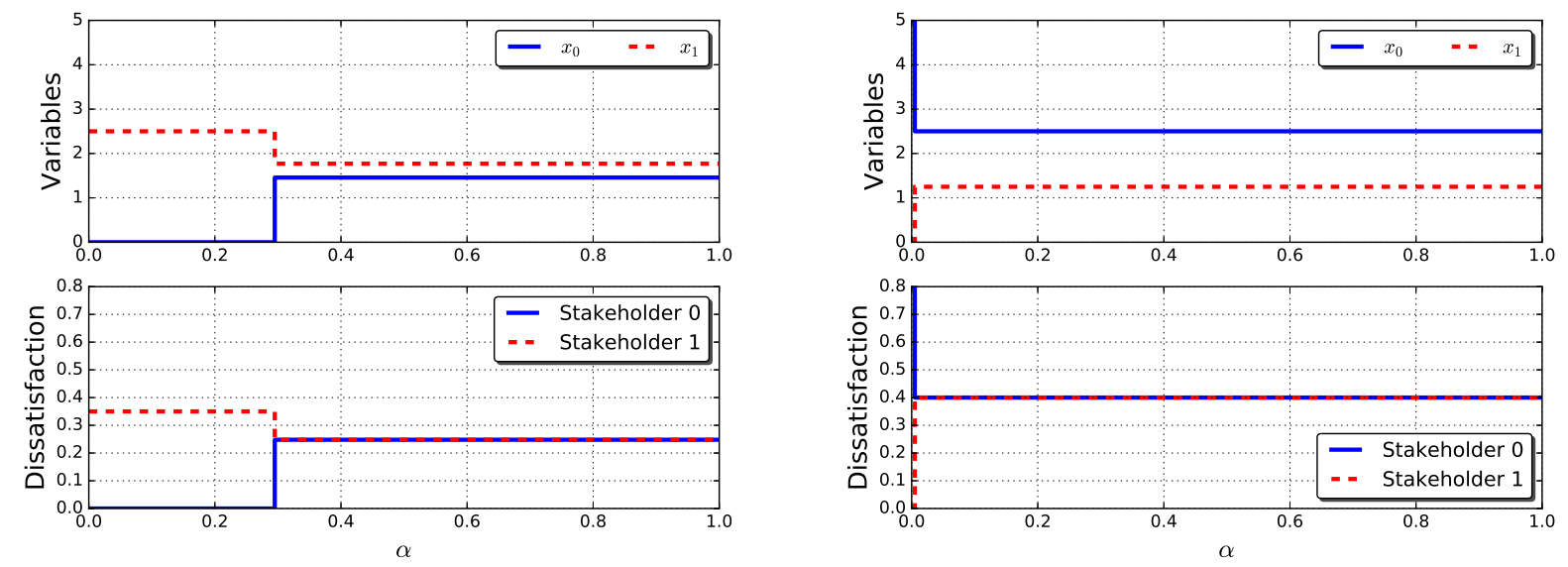

(a) Using pessimistic nadir point $[5,5]$

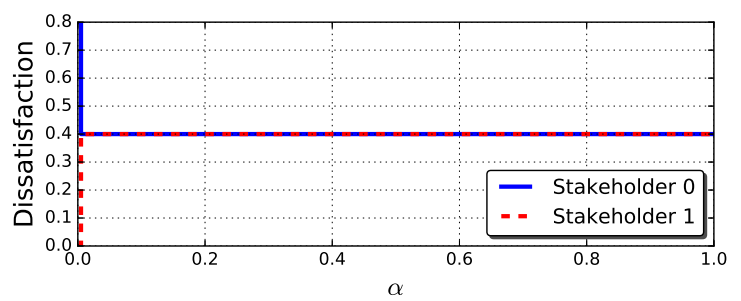

(b) Using alternate nadir point $[5,5 / 2]$

Figure 2: Solutions of CVaR problem for Example 1 as a function of $\alpha$

- CVaR only locates two points on the Pareto set of MOO (4.33) as we span $\alpha \in[0,1]$. This illustrates that CVaR only selects certain elements of the Pareto set (i.e., it does not cover the entire set). The selected elements, however, are compromise solutions that have clear interpretations from a dissatisfaction stand-point.

- The transition to a compromise solution with equal stakeholder dissatisfaction occurs at a much smaller $\alpha$ value using the alternate nadir point. This implies that consensus is reached faster by using a less pessimistic nadir point because trade-offs are evaluated on a smaller space.

\subsubsection{Example 2}

Consider the three-objective problem:

$$
\begin{array}{ll}
\min _{x} & \left(x_{0}, x_{1}, x_{2}\right) \\
\text { s.t. } & x_{0}+x_{1}+x_{2} \geq 1 \\
& 0 \leq x_{0}, x_{1}, x_{2} \leq \frac{2}{3}
\end{array}
$$

The ideal stakeholder solution $x^{*}$ for weights $\left(w_{0}, w_{1}, w_{2}\right)$ is given by:

$$
x^{*}:= \begin{cases}x_{0}=2 / 3, x_{1}=1 / 3, x_{2}=0 & \text { if } w_{0}<w_{1}<w_{2} \\ x_{0}+x_{1}=1, x_{2}=0 & \text { if } w_{0}=w_{1}<w_{2} \\ x_{0}=2 / 3, x_{1}+x_{2}=1 / 3 & \text { if } w_{0}<w_{1}=w_{2} \\ x_{0}+x_{1}+x_{2}=1 & \text { if } w_{0}=w_{1}=w_{2} .\end{cases}
$$

The objectives correspond to the actual variables (i.e., $f_{0}(x)=x_{0}, f_{1}(x)=x_{1}$, and $f_{2}(x)=x_{2}$ ) and their values lie in $[0,1]$ (no scaling is necessary). This problem also has the property that any Pareto solution lies on the plane $x_{0}+x_{1}+x_{2}=1$. We identified Pareto solutions using four sample sets of 
10 stakeholders and four sample sets of 500 stakeholders. The sets are denoted as A,B, C, and D. For each stakeholder, the weight vector $\mathbf{w}_{j}$ was drawn from a uniform distribution and the weights were normalized to satisfy (2.6). From Figure 3 we show paths that trace the evolution of the solution of the CVaR problem for equally spaced values of $\alpha \in[0,1]$. The distribution of stakeholder dissatisfactions are shown in Figure 4 for $\alpha=\{0,1 / 2,1\}$. We make the following observations:

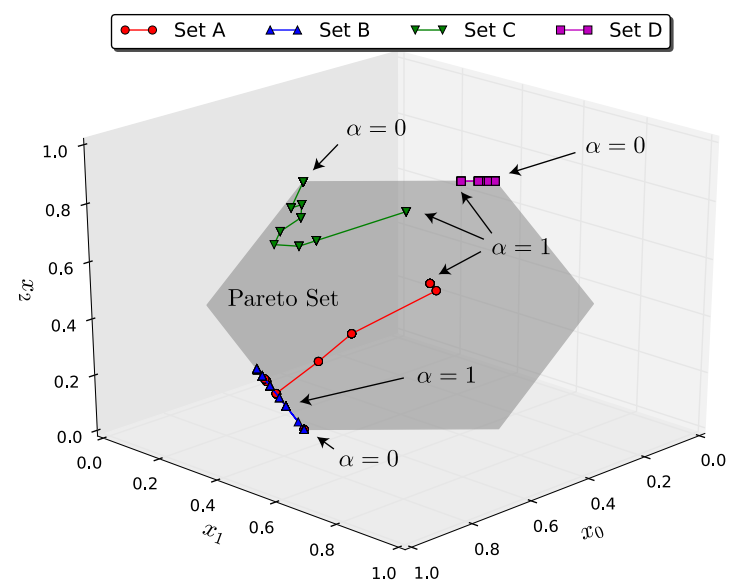

(a) Four Samples of 10 Stakeholders

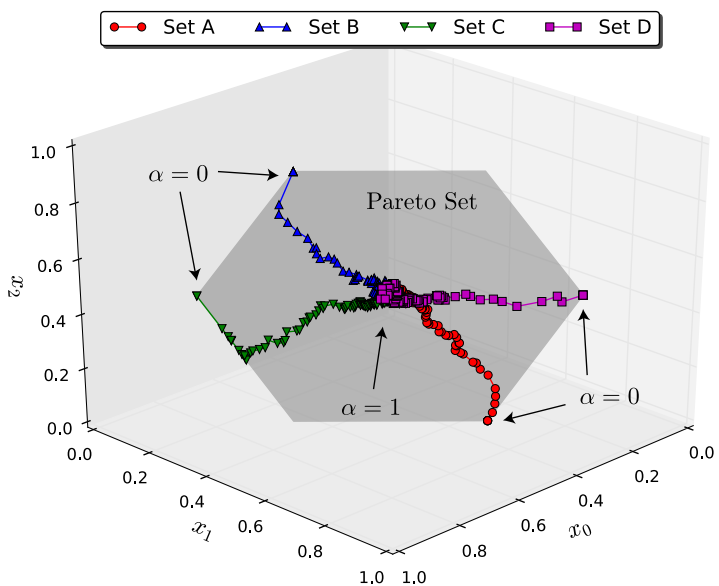

(b) Four Samples of 500 Stakeholders

Figure 3: Solutions of CVaR problem for $\alpha \in[0,1]$ for different sets of stakeholders. The verticies of the Pareto set correspond to the first condition of (4.38).

- As predicted by Theorem 1, all points on Figure 3 line on the plane $x_{0}+x_{1}+x_{2}=1$, which defines the Pareto set.

- In Figure 3 we illustrate solution behavior with increasing stakeholder population. For a small stakeholder population the CVaR solutions change significantly with different stakeholder samples. As we increase the population size we see that the CVaR solutions get closer to each other and converge at $\alpha=1$. This indicates that, as the stakeholder population increases, there is less variability in the solution that minimizes the largest dissatisfaction. Thus for this problem we can conclude that for a sufficiently large sample the worst-case compromise solution is insensitive to changes in the stakeholder sample. Depending on the application, this could undesirable because it would imply that a solution is dominated by a single stakeholder. Consequently, it would be desired to explore other compromise solutions provided by CVaR with smaller $\alpha$ values.

- In Figure 4 we illustrate that adjusting $\alpha$ changes the shape of the dissatisfaction distribution. When $\alpha=0$ (minimize mean dissatisfaction), the distribution of stakeholder dissatisfactions is skewed to the left, which indicates that the preferences of a few are sacrificed to improve the satisfaction of the many. We indicate the location of the median of the distribution instead of the mean because this gives us an indication of where half of the stakeholder population is. As $\alpha$ increases, the largest dissatisfaction decreases but the median of the dissatisfactions increases. 


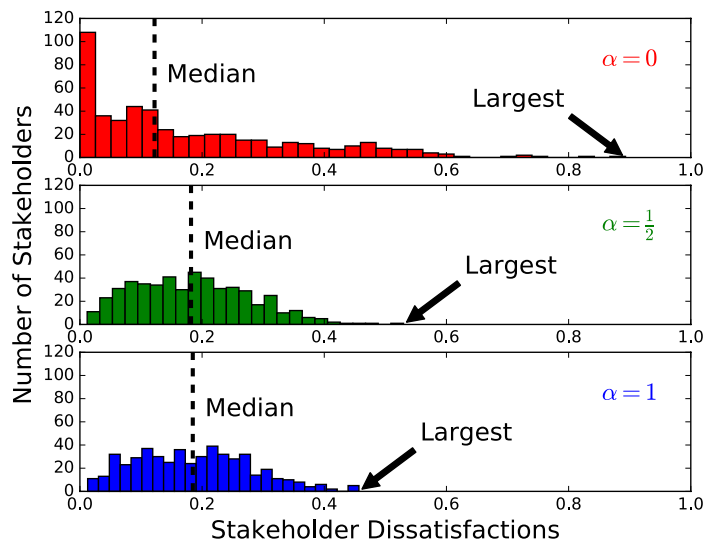

Figure 4: Dissatisfaction histograms for CVaR solutions (stakeholder set A).

For $\alpha=1$ (minimize worst dissatisfaction), the largest dissatisfaction is approximately half of that obtained with $\alpha=0$ (minimize average dissatisfaction) but the distribution is skewed to the right. The solution for $\alpha=1 / 2$ strikes a balance between the mean and the worst-case solutions.

We note that Property 1 states that CVaR is monotone in $\alpha$ for a fixed decision $x$. CVaR, however, cannot be guaranteed to be monotone as we allow the compromise solutions to change with $\alpha$. This observation can be corroborated in Table 1, which shows CVaR compromise solutions for different values of $\alpha$ and for a given stakeholder set. The trend indicates that the objective values can bounce around in an unpredictable manner as we move $\alpha$. This illustrates that identifying compromise solutions in the presence of multiple stakeholders is nontrivial and requires of optimization approaches as the one proposed here.

\section{Case Study: Facility Location Selection}

We now present a facility location problem to demonstrate the capabilities provided by the CVaR framework. The goal in this problem is to locate biowaste processing facilities in a geographical region in such a way that we: i) minimize the distance between facilities and biowaste sources (to minimize transportation), ii) maximize the distance between facilities and urban areas to avoid safety and 
Table 1: CVaR solutions for varying values of $\alpha$ (stakeholder set C).

$$
\begin{array}{cc}
\alpha=0.00 & \mathbf{f}\left(x^{*}\right)=(0.3333,0.0000,0.6667) \\
\ldots & \ldots \\
\alpha=0.43 & \mathbf{f}\left(x^{*}\right)=(0.3333,0.0000,0.6667) \\
\alpha=0.44 & \mathbf{f}\left(x^{*}\right)=(0.3901,0.0144,0.5955) \\
\ldots & \ldots \\
\alpha=0.55 & \mathbf{f}\left(x^{*}\right)=(0.3672,0.0283,0.6045) \\
\alpha=0.56 & \mathbf{f}\left(x^{*}\right)=(0.3865,0.0445,0.5689) \\
\ldots & \ldots \\
\alpha=0.64 & \mathbf{f}\left(x^{*}\right)=(0.4411,0.0273,0.5316) \\
\alpha=0.65 & \mathbf{f}\left(x^{*}\right)=(0.4694,0.0345,0.4961) \\
\ldots & \ldots \\
\alpha=0.80 & \mathbf{f}\left(x^{*}\right)=(0.3911,0.1023,0.5066) \\
\alpha=0.81 & \mathbf{f}\left(x^{*}\right)=(0.1961,0.2194,0.5845) \\
\ldots & \ldots \\
\alpha=1.00 & \mathbf{f}\left(x^{*}\right)=(0.1961,0.2194,0.5845)
\end{array}
$$

health concerns, iii) maximize the distance between facilities and water sheds to avoid contamination, and iv) minimize capital costs. This problem structure can also be used for other infrastructure placement applications, such as wind farms, wastewater treatment facilities, landfills, roadways, and so on. In all of these problems there are multiple conflicting objectives and stakeholders. In particular, the $\mathrm{CVaR}$ framework enables location of biowaste processing facilities that capture priorities of community groups, farmers, state and local administrations, federal agencies, investors, and other stakeholders want to understand some health effects as consequence of these facilities and their supply chain.

\subsection{Mathematical Model}

Let $\mathcal{F}, \mathcal{U}, \mathcal{W}$ and $\mathcal{C}$ represent the sets of farms (generating biowaste), urban centers, water sheds, and candidate facility locations, respectively. For each element $i$ of these sets, there is a corresponding spatial coordinate vector $\mathbf{z}_{i}$. The objectives are total distance from farms to facilities (denoted as $f_{0}$ ), weighted distance from each water shed and urban center to the nearest facility (denoted as $f_{1}$ and $f_{2}$, respectively), and capital costs (denoted as $f_{3}$ ). Each farm is assumed to produce one unit of biowaste, and small and large facilities are capable of processing $n^{S}$ and $n^{L}$ units of biowaste, 
respectively. The network connecting farms to facilities is modeled as follows:

$$
\begin{gathered}
\sum_{j \in \mathcal{C}} \tau_{i, j}=1, \quad i \in \mathcal{F} \\
\sum_{i \in \mathcal{F}} \tau_{i, j} \leq n^{S} y_{j}^{S}+n^{L} y_{j}^{L}, \quad j \in \mathcal{C} \\
y_{j}^{S}+y_{j}^{L} \leq 1, \quad j \in \mathcal{C} \\
y_{j}^{S}, y_{j}^{L} \in\{0,1\}, \quad j \in \mathcal{C} \\
0 \leq \tau \leq 1 \\
f_{0}=\sum_{i \in \mathcal{F}} \sum_{j \in \mathcal{C}} \tau_{i, j}\left\|\mathbf{z}_{i}-\mathbf{z}_{j}\right\|_{2},
\end{gathered}
$$

where $\tau_{i, j}$ represents the amount of biowaste transferred from farm $i$ to candidate facility location $j$. Integer variables $y_{j}^{S}$ and $y_{j}^{L}$ represent the decision to construct a small or large facility at candidate location $j$. The farm to facility objective is calculated using the network flows and farm and facility locations.

The other two distance objectives are obtained by computing the total weighted distance from each urban center and water shed to the nearest facility:

$$
\begin{array}{cc}
r_{i} \leq\left\|\mathbf{z}_{i}-\mathbf{z}_{j}\right\|_{2}\left(\alpha^{S} y_{j}^{S}+\alpha^{L} y_{j}^{L}\right)+M \bar{\alpha}\left(1-y_{j}^{S}-y_{j}^{L}\right), & i \in \mathcal{U}, j \in \mathcal{C} \\
r_{i} \leq\left\|\mathbf{z}_{i}-\mathbf{z}_{j}\right\|_{2}\left(\beta^{S} y_{j}^{S}+\beta^{L} y_{j}^{L}\right)+M \bar{\beta}\left(1-y_{j}^{S}-y_{j}^{L}\right), \quad i \in \mathcal{W}, j \in \mathcal{C} & \\
r_{i} \geq 0, \quad i \in \mathcal{U} \cup \mathcal{W} & \\
f_{1}=\sum_{i \in \mathcal{U}} r_{i}, \quad f_{2}=\sum_{i \in \mathcal{W}} r_{i}, &
\end{array}
$$

where $\alpha^{S}, \alpha^{L}, \beta^{S}, \beta^{L}$ are constants representing the impact of small versus large facilities on the distance to urban centers and water shed metrics. The variable $r_{i}$ represents the largest distance from a urban center or water shed $i$ to the nearest facility. Parameter $M$ is set to the largest possible distance and we define $\bar{\alpha}=\max \left(\alpha^{S}, \alpha^{L}\right)$ and $\bar{\beta}=\max \left(\beta^{S}, \beta^{L}\right)$. Capital cost is computed as follows:

$$
f_{3}=\sum_{j \in \mathcal{C}}\left(c^{S} y_{j}^{S}+c^{L} y_{j}^{L}\right)
$$

where $c^{S}$ and $c^{L}$ are the costs of small and large facilities, respectively.

\subsection{Computational Results}

Locational data for 12 farms, 2 urban centers, 3 watersheds and 30 candidate facility locations were generated from a uniform distribution. Similarly, the priorities of 50 stakeholders were generated from a uniform normal distribution and normalized. We solved the CVaR problem for the four scaled objectives $f_{0},-f_{1},-f_{2}$ and $f_{3}$ using 101 evenly spaced values of $\alpha$ covering the range $[0,1]$. We compare results using scaling obtained from the standard (pessimistic) nadir point and the alternate nadir point. The results are summarized Table 2 . The compromise solutions computed with the alternate nadir point for varying values of $\alpha$ are visualized in Figure 5. In Figure 6 we compare 
the solutions obtained by minimizing distance between farms and facilities $\left(f_{1}\right)$ using formulations (2.2)-(2.3) and (2.9). We make the following observations:

- In all of the compromise solutions generated with the alternate nadir point, shown in Figure 5, only one small facility and one large facility were installed. The location of the small facility is constant across all of the solutions while the location of the large facility changes with $\alpha$. The four locations for the large facility seen as $\alpha$ is traced from zero to one are accented with ellipses in Figure 5. It is thus clear that, with the collected stakeholder preferences sample, a debate should be focused on four candidate locations for the large processing facility, and not the other 26 candidate locations or the location of the small processing facility. This illustrates that the CVaR framework can facilitate discussions by better informing stakeholders seeking to evaluate a wide range of alternatives.

- As in Example 1, this case study is prone to pessimistic nadir points. The optimization results for minimizing distance between farms and facilities, computed by (2.2) and (2.9), are compared in Figure 6. With the standard nadir point, defined by (2.2), a solution with a large facility at every candidate location is selected. This is because $f_{1}, f_{2}$ and $f_{3}$ are not considered in the objective, and thus there is no motivation to build fewer facilities. Similar extreme solution behavior is apparent in Table 2, and the explored solution space (between the utopia and nadir points) is wide. With the alternate nadir point of (2.9), a less pessimistic solution is generated and this narrows down the range. In particular, the standard nadir point is $(9.68,0.00,0.00$, 255.00), whereas the alternate point is $(8.98,0.54,2.90,18.5)$.

- From Table 2 we see that using the pessimistic nadir point results in large trade-offs for the first three objectives as $\alpha$ spans [0,1]. With the alternate nadir point we only observe trade-offs between the first two objectives as $\alpha$ changes. As indicated by Theorem 1 we have that the CVaR compromise solutions are Pareto solutions of MOO for all $\alpha \in[0,1]$, regardless of the nadir point used (which sets the objective scaling). Scaling is important, however, and impacts the portion of the Pareto set explored by the CVaR problem. For instance, when using the alternate nadir point, the CVaR compromise solutions for $\alpha \in[0.00,0.49]$ and $\alpha \in[0.72,0.86]$ are identical to the solution obtained by the individual minimization of $f_{4}$ (capital cost). This illustrates that the alternate nadir point is Pareto optimal (from Proposition 2). This also illustrates that the use of the alternate nadir solution narrows down the decision space and facilitates decision-making.

- Figure 7 illustrates the solutions for the four stakeholder with the strongest biases on individual objectives. Interestingly, none of these solutions match the compromise solutions generated by $\mathrm{CVaR}$ minimization. This illustrates both the diversity of the preferred solutions for stakeholders and that the $\mathrm{CVaR}$ framework finds compromise solutions and facilitates targeted discussions. 


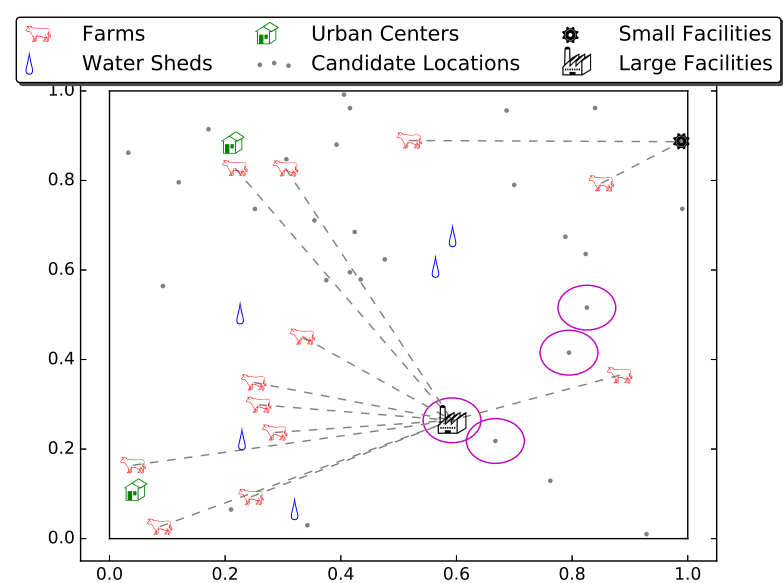

(a) $\alpha \in[0,0.49]$

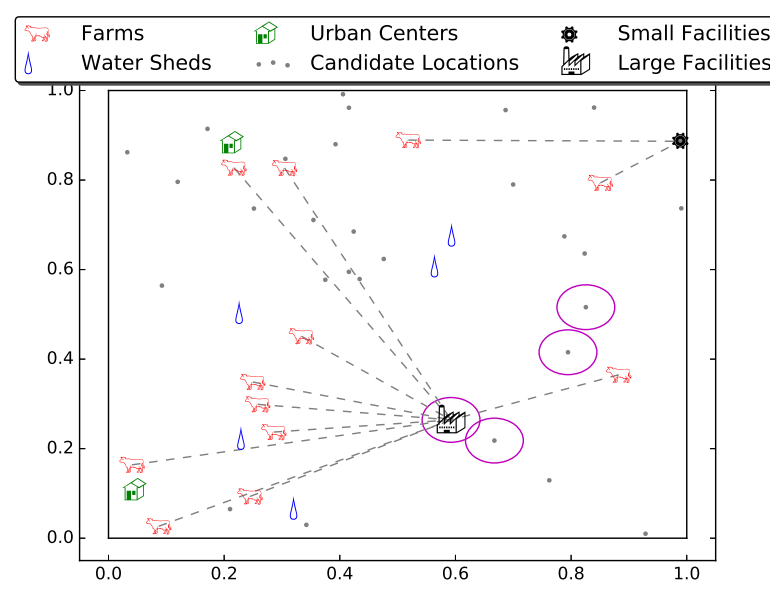

(c) $\alpha \in[0.72,0.86]$

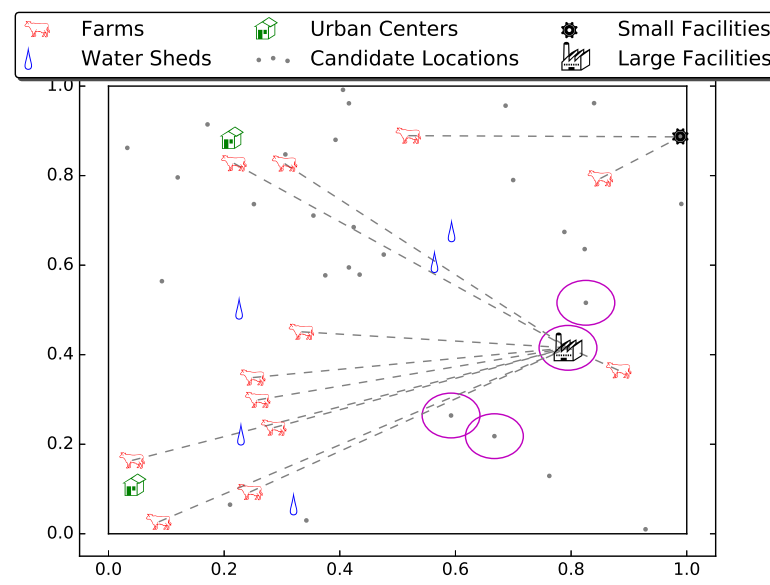

(e) $\alpha \in[0.95,0.97]$

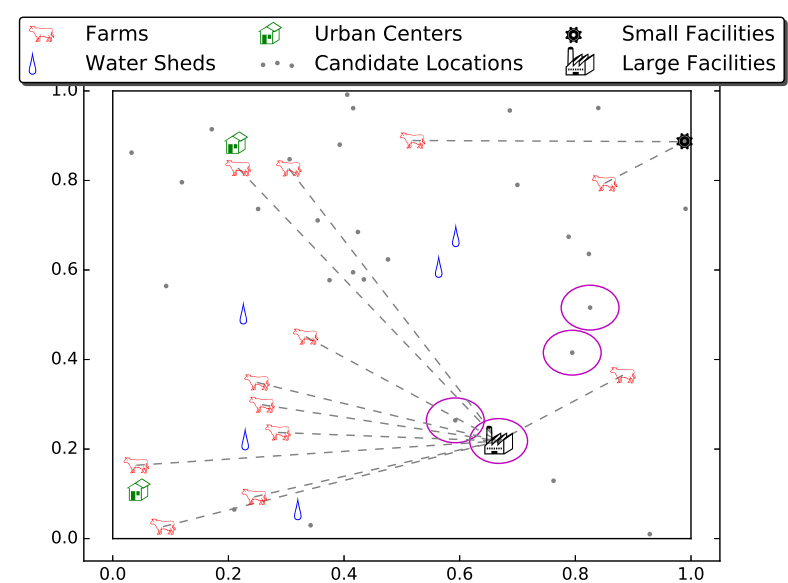

(b) $\alpha \in[0.50,0.71]$

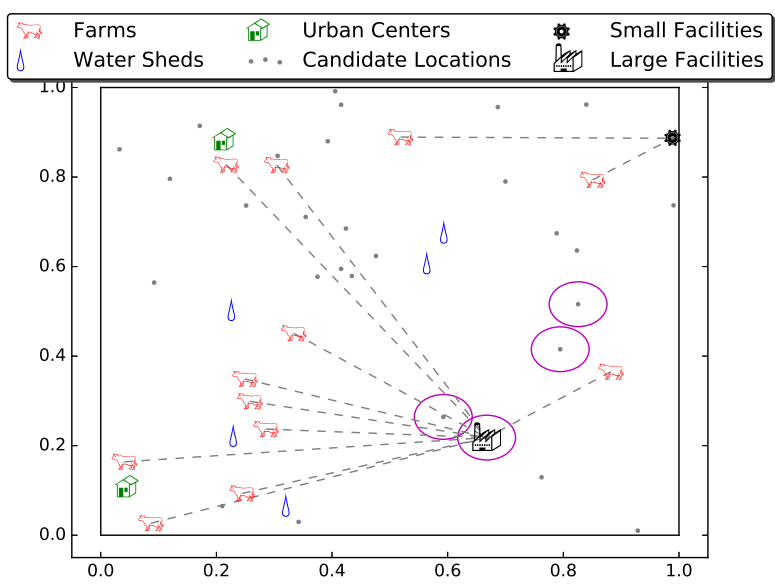

(d) $\alpha \in[0.87,0.94]$

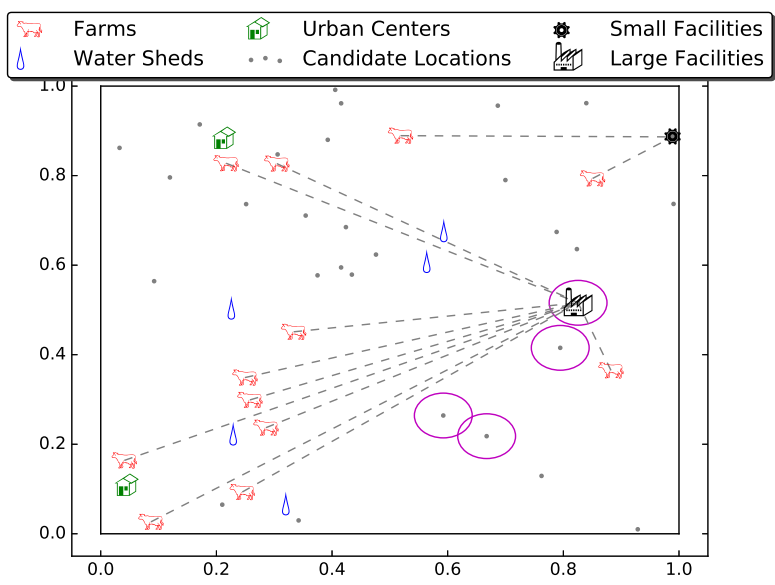

(f) $\alpha \in[0.98,1]$

Figure 5: CVaR compromise solutions for biowaste case study. 
Table 2: Solutions for biowaste facility location problem.

\begin{tabular}{|c|c|c|c|c|c|c|}
\hline \multirow[b]{2}{*}{ Name } & \multicolumn{4}{|c|}{ Objective Values } & \multicolumn{2}{|c|}{ No. of Facilities } \\
\hline & $f_{0}$ & $f_{1}$ & $f_{2}$ & $f_{3}$ & Small & Large \\
\hline \multicolumn{7}{|c|}{ Using Pessimistic Nadir Point } \\
\hline $\operatorname{Min} f_{0}$ & 1.66 & 0.0 & 0.0 & 255.0 & 0 & 30 \\
\hline $\operatorname{Max} f_{1}$ & 9.31 & 3.82 & 0.0 & 17.0 & 0 & 2 \\
\hline $\operatorname{Max} f_{2}$ & 9.68 & 0.0 & 34.08 & 17.0 & 0 & 2 \\
\hline $\operatorname{Min} f_{3}$ & 6.79 & 0.0 & 0.0 & 10.5 & 1 & 1 \\
\hline Utopia & 1.66 & 3.82 & 34.08 & 10.5 & - & - \\
\hline Nadir & 9.68 & 0.0 & 0.0 & 255.0 & - & - \\
\hline$\alpha \in[0.00,0.59]$ & 8.98 & 3.82 & 33.40 & 17.0 & 0 & 2 \\
\hline$\alpha \in[0.60,0.63]$ & 8.57 & 3.53 & 32.84 & 17.0 & 0 & 2 \\
\hline$\alpha \in[0.64,0.74]$ & 7.80 & 3.33 & 29.95 & 17.0 & 0 & 2 \\
\hline$\alpha \in[0.75,0.89]$ & 6.40 & 2.99 & 24.69 & 17.0 & 0 & 2 \\
\hline$\alpha=0.9$ & 6.13 & 2.88 & 22.73 & 17.0 & 0 & 2 \\
\hline$\alpha \in[0.91,0.92]$ & 5.99 & 2.70 & 23.45 & 17.0 & 0 & 2 \\
\hline$\alpha \in[0.93,1.00]$ & 5.67 & 2.82 & 20.85 & 17.0 & 0 & 2 \\
\hline \multicolumn{7}{|c|}{ Using Alternate Nadir Point } \\
\hline $\operatorname{Min} f_{0}$ & 1.66 & 0.54 & 2.90 & 18.5 & 5 & 1 \\
\hline $\operatorname{Max} f_{1}$ & 8.98 & 3.82 & 33.39 & 17.0 & 0 & 2 \\
\hline $\operatorname{Max} f_{2}$ & 8.96 & 3.60 & 34.08 & 17.0 & 0 & 2 \\
\hline $\operatorname{Min} f_{3}$ & 5.08 & 2.59 & 7.76 & 10.5 & 1 & 1 \\
\hline Utopia & 1.66 & 3.82 & 34.08 & 10.5 & - & - \\
\hline Nadir & 8.98 & 0.54 & 2.90 & 18.5 & - & - \\
\hline$\alpha \in[0.00,0.49]$ & 5.08 & 2.59 & 7.76 & 10.5 & 1 & 1 \\
\hline$\alpha \in[0.50,0.71]$ & 5.69 & 2.82 & 7.76 & 10.5 & 1 & 1 \\
\hline$\alpha \in[0.72,0.86]$ & 5.08 & 2.59 & 7.76 & 10.5 & 1 & 1 \\
\hline$\alpha \in[0.87,0.94]$ & 5.69 & 2.82 & 7.76 & 10.5 & 1 & 1 \\
\hline$\alpha \in[0.95,0.97]$ & 6.43 & 3.11 & 7.76 & 10.5 & 1 & 1 \\
\hline$\alpha \in[0.98,1.00]$ & 6.86 & 3.12 & 7.76 & 10.5 & 1 & 1 \\
\hline
\end{tabular}




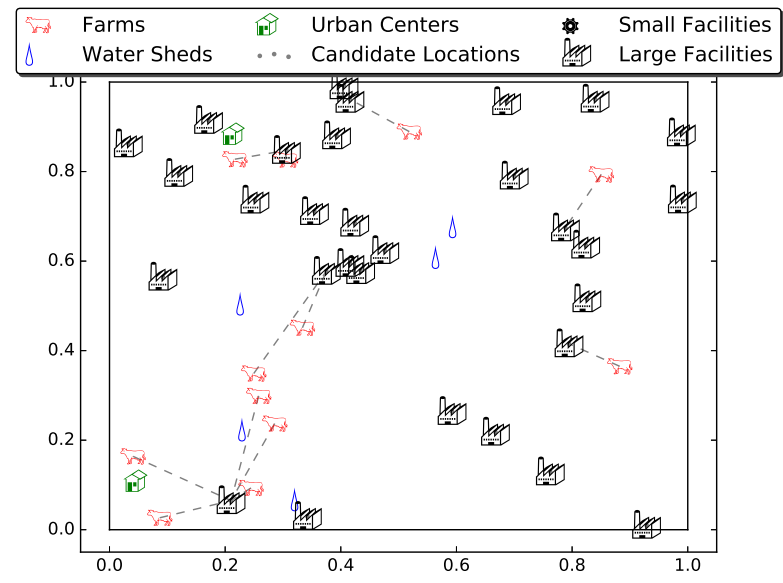

(a) Solution for Min $f_{0}$ per (2.2)

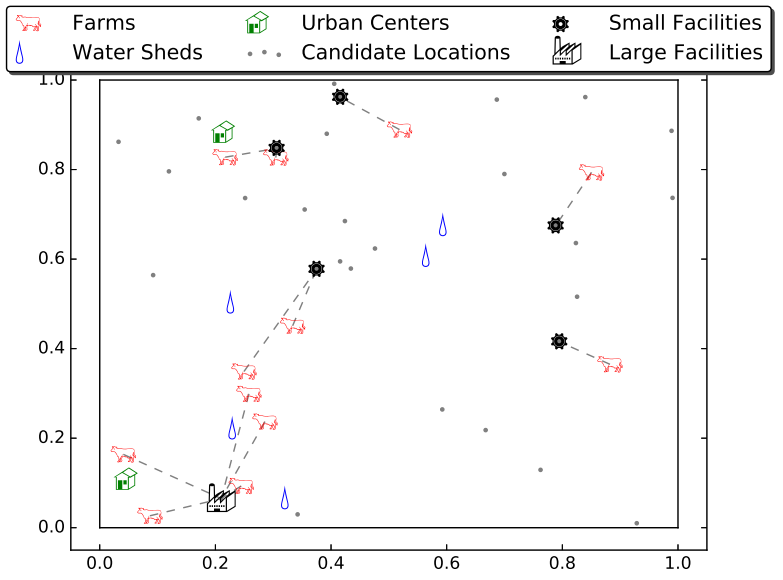

(b) Solution for Min $f_{0}$ per (2.9)

Figure 6: Comparison of single objective solutions for farm-to-facility distance objective $\left(f_{0}\right)$.

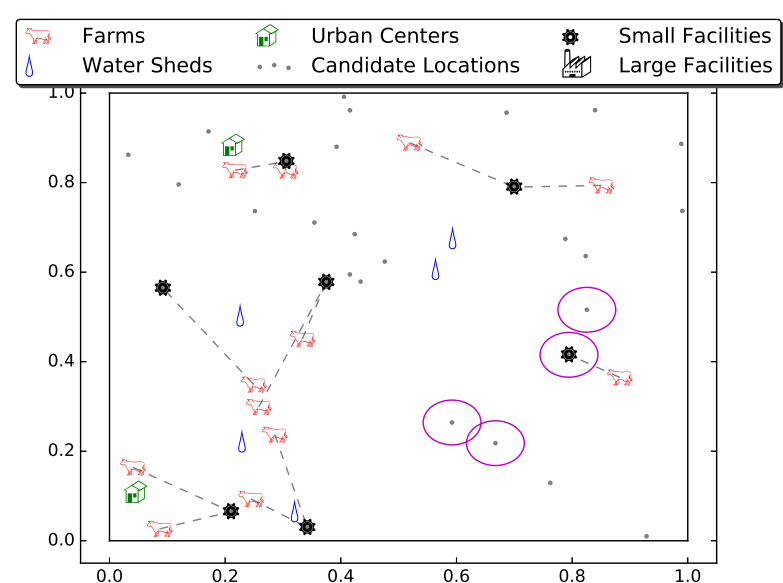

(a) Stakeholder biased towards $f_{0}$

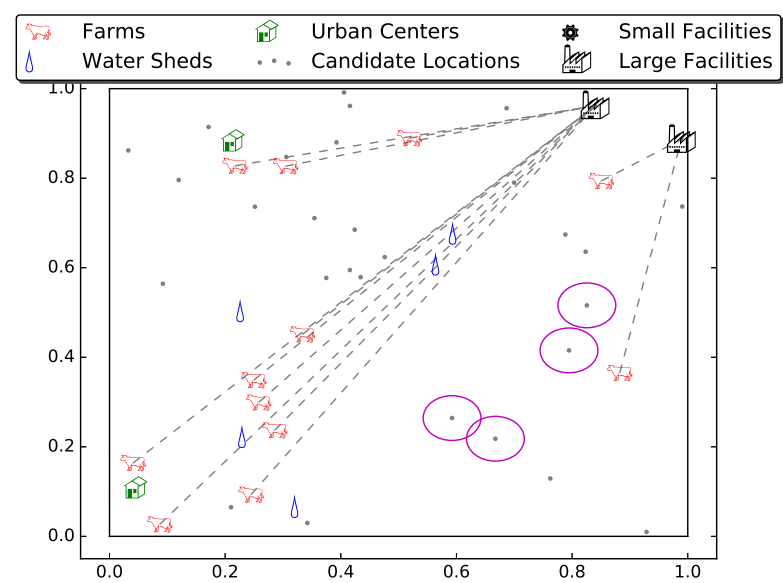

(c) Stakeholder biased towards $f_{2}$

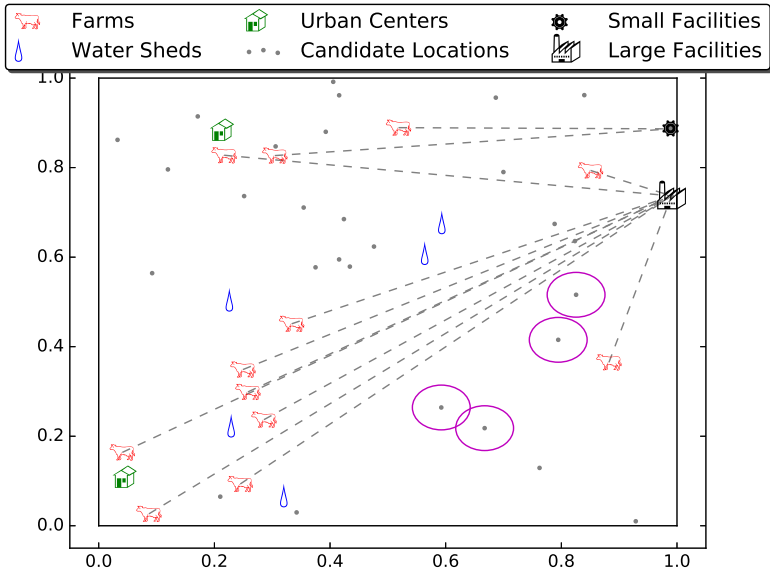

(b) Stakeholder biased towards $f_{1}$

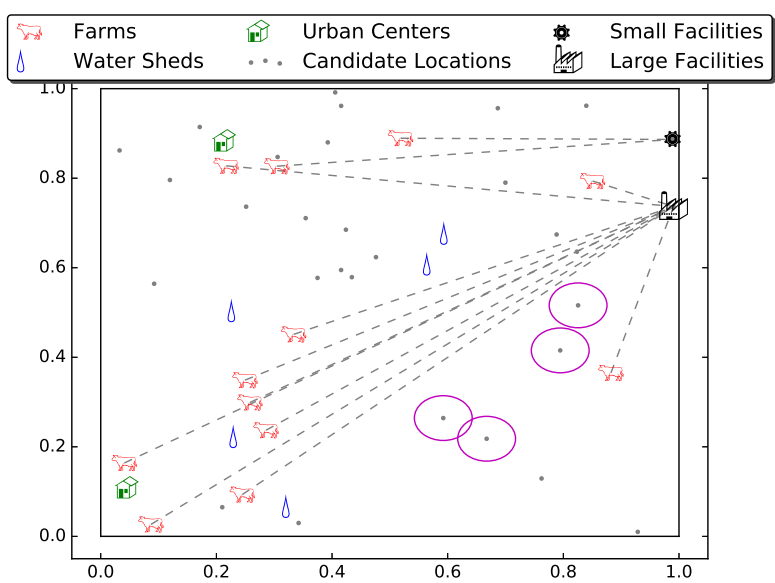

(d) Stakeholder biased towards $f_{3}$

Figure 7: Comparison of solutions for stakeholders with strongly biased preferences. 


\section{Conclusions and Future Work}

We presented a conditional-value-at-risk (CVaR) framework for multi-stakeholder decision-making. In the proposed setting we factor in stakeholder priorities on multiple objectives to construct dissatisfaction functions and we interpret such dissatisfactions as random variables. This allows us to provide statistical interpretations to the resulting compromise solutions. We use a geometric interpretation of $\mathrm{CVaR}$ to prove that any compromise solution obtained with the proposed framework is a Pareto optimal solution for the original multi-objective optimization problem. In this sense, our approach is a generalization of previous approaches that either minimize average stakeholder dissatisfaction or the worst-case stakeholder dissatisfaction. We also demonstrate that the framework offers a way to interpret compromise solutions in terms of stakeholder dissatisfactions, which can facilitate discussions. The framework also provides a mechanism to explore and quantify the effect of opinions on final decisions. The framework is targeted at problems with many competing objectives and stakeholder preferences, for which computing the entire Pareto set is not practical.

The framework may be extended by refining the conditions for the weights of Theorem 1 that guarantee Pareto optimality. In addition, it is possible to perform post-solution checks to guarantee that a compromise solution is indeed Pareto optimal [71,43]. The proposed framework may also be extended to consider alternate coherent risk measures and it is also possible to consider metrics with direct fairness interpretations. We also highlight that the proposed framework requires global optimal solutions for the stakeholder problems and for the CVaR minimization problem. It is thus necessary to extend the framework to allow for local optimal solutions and to provide suitable interpretations.

\section{Acknowledgments}

Victor M. Zavala acknowledges funding from the U.S. Department of Energy Early Career program and thanks Sanjay Mehrotra and Kibaek Kim for technical discussions that motivated this work.

\section{References}

[1] G. K. Al-Sharrah, I. Alatiqi, a. Elkamel, and E. Alper. Planning an Integrated Petrochemical Industry with an Environmental Objective. Industrial E Engineering Chemistry Research, 40(9):21032111, 2001.

[2] V. Andiappan, A. S. Y. Ko, V. W. S. Lau, L. Y. Ng, R. T. L. Ng, N. G. Chemmangattuvalappil, and D. K. S. Ng. Synthesis of Sustainable Integrated Biorefinery via Reaction Pathway Synthesis: Economic, Incremental Enviromental Burden and Energy Assessment with Multiobjective Optimization. AIChE Journal, 61(1):132-146, 2015.

[3] S. Bairamzadeh, M. S. Pishvaee, and M. Saidi-Mehrabad. Multiobjective Robust Possibilistic Programming Approach to Sustainable Bioethanol Supply Chain Design Under Multiple Uncertainties. Industrial \& Engineering Chemistry Research, 55:237-256, 2016. 
[4] B. R. Bakshi and J. Fiksel. The quest for sustainability: Challenges for process systems engineering. AIChE Journal, 49(6):1350-1358, 2003.

[5] H. S. Bamufleh, J. M. Ponce-Ortega, and M. M. El-Halwagi. Multi-objective optimization of process cogeneration systems with economic, environmental, and social tradeoffs. Clean Technologies and Environmental Policy, 15(1):185-197, 2013.

[6] A. Bernardi, S. Giarola, and F. Bezzo. Spatially Explicit Multiobjective Optimization for the Strategic Design of First and Second Generation Biorefineries Including Carbon and Water Footprints. Industrial \& Engineering Chemistry Research, 52:7170-7180, 2013.

[7] V. Bhaskar, S. K. Gupta, and A. K. Ray. Multiobjective optimization of an industrial wiped-film pet reactor. AIChE Journal, 46(5):1046-1058, 2000.

[8] F. E. Boran, S. Genç, M. Kurt, and D. Akay. A multi-criteria intuitionistic fuzzy group decision making for supplier selection with topsis method. Expert Systems with Applications, 36(8):1136311368, 2009.

[9] R. Brunet, G. Guill, and L. Jim. Cleaner Design of Single-Product Biotechnological Facilities through the Integration of Process Simulation, Multiobjective Optimization, Life Cycle Assessment, and Principal Component Analysis. Industrial E Engineering Chemistry Research, 51:410424, 2012.

[10] O. Burgara-Montero, A. A. El-Baz, J. M. Ponce-Ortega, and M. M. El-Halwagi. Optimal design of a distributed treatment system for increasing dissolved oxygen in watersheds through selfrotating discs. ACS Sustainable Chemistry and Engineering, 1(10):1267-1279, 2013.

[11] L. Čuček, J. J. Klemeš, P. S. Varbanov, and Z. Kravanja. Dealing with high-dimensionality of criteria in multiobjective optimization of biomass energy supply network. Industrial and Engineering Chemistry Research, 52(22):7223-7239, 2013.

[12] I. Das and J. E. Dennis. Normal-boundary intersection: A new method for generating the pareto surface in nonlinear multicriteria optimization problems. SIAM Journal on Optimization, 8(3):631657, 1998.

[13] F. A. Diaz-Alvarado. An example of Pareto dominance for dimensionality reduction in multiobjective optimization. Computers and Chemical Engineering, 79:135-136, 2015.

[14] U. M. Diwekar. Greener by Design. Environmental Science and Technology, 37(23):5432-5444, 2003.

[15] U. M. Diwekar and Y. N. Shastri. Green process design, green energy, and sustainability: A systems analysis perspective. Computers and Chemical Engineering, 34(9):1348-1355, 2010.

[16] R. F. Dyer and E. H. Forman. Group decision support with the analytic hierarchy process. Decision support systems, 8(2):99-124, 1992. 
[17] A. M. El-Halwagi, C. Rosas, J. M. Ponce-Ortega, A. Jiménez-Gutiérrez, M. S. Mannan, and M. M. El-Halwagi. Multiobjective optimization of biorefineries with economic and safety objectives. AIChE Journal, 59(7):2427-2434, 2013.

[18] J. C. Eslick and D. C. Miller. A multi-objective analysis for the retrofit of a pulverized coal power plant with a $\mathrm{CO} 2$ capture and compression process. Computers and Chemical Engineering, 35(8):1488-1500, 2011.

[19] B. H. Gebreslassie, R. Waymire, and F. You. Sustainable Design and Synthesis of AlgaeBased Biorefinery for Simultaneous Hydrocarbon Biofuel Production and Carbon Sequestration. AIChE Journal, 59(5):1599-1621, 2013.

[20] B. H. Gebreslassie, Y. Yao, and F. You. Design Under Uncertainty of Hydrocarbon Biorefinery Supply Chains: Multiobjective Stochastic Programming Models, Decomposition Algorithm, and a Comparison Between CVaR and Downside Risk. AIChE Journal, 58(7):2155-2179, 2012.

[21] A. Geraili and J. A. Romagnoli. A Multiobjective Optimization Framework for Design of Integrated Biorefineries Under Uncertainty. AIChE Journal, 61(10):3208-3222, 2015.

[22] S. Giarola, A. Zamboni, and F. Bezzo. Spatially explicit multi-objective optimisation for design and planning of hybrid first and second generation biorefineries. Computers and Chemical Engineering, 35(9):1782-1797, 2011.

[23] J. González-Pachón and C. Romero. Distance-based consensus methods: a goal programming approach. Omega, 27(3):341-347, 1999.

[24] I. E. Grossmann and G. Guillén-Gosálbez. Scope for the application of mathematical programming techniques in the synthesis and planning of sustainable processes. Computers and Chemical Engineering, 34(9):1365-1376, 2010.

[25] G. Guillén-Gosálbez. A novel MILP-based objective reduction method for multi-objective optimization: Application to environmental problems. Computers and Chemical Engineering, 35:14691477, 2011.

[26] G. Guillén-Gosálbez and I. E. Grossmann. Optimal design and planning of sustainable chemical supply chains under uncertainty. AIChE Journal, 55(1):99-121, 2009.

[27] C. G. Gutiérrez-Arriaga, M. Serna-González, J. M. Ponce-Ortega, and M. M. El-Halwagi. Sustainable Integration of Algal Biodiesel Production with Steam Electric-Power Plants for Greenhouse Gas Mitigation. ACS Sustainable Chemistry E Engineering, 2:1388-1403, 2014.

[28] H. Halsall-Whitney and J. Thibault. Multi-objective optimization for chemical processes and controller design: Approximating and classifying the Pareto domain. Computers and Chemical Engineering, 30(6-7):1155-1168, 2006.

[29] R. J. Hanes and B. R. Bakshi. Sustainable Process Design by the Process to Planet. AIChE Journal, 61(10):3320-3331, 2015. 
[30] J. Hu and S. Mehrotra. Robust and stochastically weighted multiobjective optimization models and reformulations. Operations Research, 60(4):936-953, 2012.

[31] C. D. Immanuel and F. J. Doyle. Hierarchical multiobjective strategy for particle-size distribution control. AIChE Journal, 49(9):2383-2399, 2003.

[32] J. Iwaro, A. Mwasha, R. G. Williams, and R. Zico. An integrated criteria weighting framework for the sustainable performance assessment and design of building envelope. Renewable and Sustainable Energy Reviews, 29:417-434, 2014.

[33] Z. Jia and M. G. Ierapetritou. Generate Pareto optimal solutions of scheduling problems using normal boundary intersection technique. Computers and Chemical Engineering, 31(4):268-280, 2007.

[34] C. Jimenez-Gonzalez and J. M. Woodley. Bioprocesses: Modeling needs for process evaluation and sustainability assessment. Computers and Chemical Engineering, 34(7):1009-1017, 2010.

[35] A. J. Kleywegt, A. Shapiro, and T. Homem-de Mello. The sample average approximation method for stochastic discrete optimization. SIAM Journal on Optimization, 12(2):479-502, 2002.

[36] K. B. Lee, R. B. Kasat, G. B. Cox, and N.-H. L. Wang. Simulated Moving Bed Multiobjective Optimization Using Standing Wave Design and Genetic Algorithm. AIChE Journal, 54(11):2852$2871,2008$.

[37] M. Leipold, S. Gruetzmann, and G. Fieg. An evolutionary approach for multi-objective dynamic optimization applied to middle vessel batch distillation. Computers and Chemical Engineering, 33(4):857-870, 2009.

[38] W. H. Liew, M. H. Hassim, D. K. S. Ng, and N. Chemmangattuvalappil. Systematic Framework for Sustainability Assessment of Biodiesel Production: Preliminary Engineering Stage. Industrial and Engineering Chemistry Research, 54(50):12615-12629, 2015.

[39] J. Linderoth, A. Shapiro, and S. Wright. The empirical behavior of sampling methods for stochastic programming. Annals of Operations Research, 142(1):215-241, 2006.

[40] L. F. Lira-Barragan, J. M. Ponce-Ortega, M. Serna-Gonzalez, and M. M. El-Halwagi. An MINLP model for the optimal location of a new industrial plant with simultaneous consideration of economic and environmental criteria. Industrial and Engineering Chemistry Research, 50(2):953964, 2011.

[41] F. Logist, P. M. M. Van Erdeghem, and J. F. Van Impe. Efficient deterministic multiple objective optimal control of (bio)chemical processes. Chemical Engineering Science, 64(11):2527-2538, 2009.

[42] G. A. Mendoza and R. Prabhu. Multiple criteria decision making approaches to assessing forest sustainability using criteria and indicators: a case study. Forest Ecology and Management, 131(1):107-126, 2000. 
[43] K. Miettinen. Nonlinear multiobjective optimization, volume 12. Springer, 1999.

[44] K. Mitra, S. Majumdar, and S. Raha. Multiobjective dynamic optimization of a semi-batch epoxy polymerization process. Computers and Chemical Engineering, 28(12):2583-2594, 2004.

[45] D. Nagrath, W. W. Bequette, S. M. Cramer, and A. Messac. Multiobjective optimization strategies for linear gradient chromatography. AIChE Journal, 51(2):511-525, 2005.

[46] T. T. H. Nguyen, Y. Kikuchi, M. Noda, and M. Hirao. A New Approach for the Design and Assessment of Bio-based Chemical Processes toward Sustainability. Industrial E Engineering Chemistry Research, 54(20):5494-5504, 2015.

[47] S. Opricovic. A compromise solution in water resources planning. Water Resources Management, 23(8):1549-1561, 2009.

[48] S. Opricovic and G.-H. Tzeng. Compromise solution by MCDM methods: A comparative analysis of VIKOR and TOPSIS. European Journal of Operational Research, 156(2):445-455, 2004.

[49] A. I. Papadopoulos and P. Linke. Multiobjective molecular design for integrated process-solvent systems synthesis. AIChE Journal, 52(3):1057-1069, 2006.

[50] K. Pavlikov and S. Uryasev. CVaR norm and applications in optimization. Optimization Letters, 8(7):1999-2020, 2014.

[51] L. Pedraza-Segura, H. Toribio-Cuaya, and A. Flores-Tlacuahuac. Multiobjective Optimization Approach for Cellulosic Biomass Pretreatment. Industrial \& Engineering Chemistry Research, 52(15):5357-5364, 2013.

[52] T. Prihatin, M. Shuhaimi, M. I. A. Mutalib, and M. D. Bustan. Synthesis of optimum water polygeneration system in ethylene glycol production. Industrial and Engineering Chemistry Research, 52(22):7066-7073, 2013.

[53] M. G. Rojas-Torres, F. Napoles-Rivera, J. M. Ponce-Ortega, M. Serna-González, G. GuillenGosalbez, and J.-E. Laureano. Multiobjective Optimization for Designing and Operating More Sustainable Water Management Systems for a City in Mexico. AIChE Journal, 61(8):2428-2446, 2015.

[54] G. J. Ruiz-Mercado, R. L. Smith, and M. A. Gonzalez. Sustainability indicators for chemical processes: I. taxonomy. Industrial \& Engineering Chemistry Research, 51(5):2309-2328, 2012.

[55] H. Safikhani, A. Hajiloo, and M. A. Ranjbar. Modeling and multi-objective optimization of cyclone separators using CFD and genetic algorithms. Computers and Chemical Engineering, 35(6):1064-1071, 2011.

[56] B. Sankararao and S. K. Gupta. Multi-objective optimization of an industrial fluidized-bed catalytic cracking unit (FCCU) using two jumping gene adaptations of simulated annealing. Computers and Chemical Engineering, 31(11):1496-1515, 2007. 
[57] J. E. Santibanez-Aguilar, J. M. Ponce-Ortega, J. B. Gonzalez-Campos, M. Serna-Gonzalez, and M. M. El-Halwagi. Synthesis of Distributed Biorefining Networks for the Value-Added Processing of Water Hyacinth. ACS Sustainable Chemistry \& Engineering, 1:284-305, 2013.

[58] D. Sarkar, S. Rohani, and A. Jutan. Multiobjective Optimization of Semibatch Reactive Crystallization Processes. AIChE Journal, 53(5):1164-1177, 2007.

[59] C. M. Silva and E. C. Biscaia. Genetic algorithm development for multi-objective optimization of batch free-radical polymerization reactors. Computers and Chemical Engineering, 27(8-9):13291344, 2003.

[60] A. Singh and H. H. Lou. Hierarchical pareto optimization for the sustainable development of industrial ecosystems. Industrial and Engineering Chemistry Research, 45(9):3265-3279, 2006.

[61] R. L. Smith and G. J. Ruiz-Mercado. A method for decision making using sustainability indicators. Clean Technologies and Environmental Policy, 16(4):749-755, 2014.

[62] R. L. Smith and G. J. Ruiz-Mercado. A method for decision making using sustainability indicators. Clean Technologies and Environmental Policy, 16(4):749-755, 2014.

[63] R. Tudor and V. Lavric. Dual-objective optimization of integrated water/wastewater networks. Computers and Chemical Engineering, 35(12):2853-2866, 2011.

[64] P. Vaskan, G. Guillen-Gosalbez, M. Turkay, and L. Jimenez. Multiobjective optimization of utility plants under several environmental indicators using an MILP-based dimensionality reduction approach. Industrial E Engineering Chemistry Research, 53(50):19559-19572, 2014.

[65] B. Wang, B. H. Gebreslassie, and F. You. Sustainable design and synthesis of hydrocarbon biorefinery via gasification pathway: Integrated life cycle assessment and technoeconomic analysis with multiobjective superstructure optimization. Computers and Chemical Engineering, 52:55-76, 2013.

[66] A. K. Y. Yee, A. K. Ray, and G. P. Rangaiah. Multiobjective optimization of an industrial styrene reactor. Computers and Chemical Engineering, 27(1):111-130, 2003.

[67] F. You, L. Tao, D. J. Graziano, and S. W. Snyder. Optimal Design of Sustainable Cellulosic Biofuel Supply Chains: Multiobjective Optimization Coupled with Life Cycle Assessment and InputOutput Analysis. AIChE Journal, 58(4):1157-1180, 2011.

[68] F. Q. You and B. Wang. Life Cycle Optimization of Biomass-to-Liquid Supply Chains with Distributed-Centralized Processing Networks. Industrial \& Engineering Chemistry Research, 50(17):10102-10127, 2011.

[69] D. Yue, M. Slivinsky, J. Sumpter, and F. You. Sustainable design and operation of cellulosic bioelectricity supply chain networks with life cycle economic, environmental, and social optimization. Industrial and Engineering Chemistry Research, 53(10):4008-4029, 2014. 
[70] V. M. Zavala. Managing conflicts among decision-makers in multiobjective design and operations. In Sustainability in the Analysis, Synthesis and Design of Chemical Engineering Processes, To Appear, 2015.

[71] V. M. Zavala. A multiobjective optimization perspective on the stability of economic MPC. IFAC-PapersOnLine, 48(8):974 - 980, 2015.

[72] W. Zhang, C. Wang, Y. Li, P. Wang, Q. Wang, and D. Wang. Seeking sustainability: Multiobjective evolutionary optimization for urban wastewater reuse in China. Environmental Science and Technology, 48(2):1094-1102, 2014.

[73] Z. Zhang, K. Hidajat, A. K. Ray, and M. Morbidelli. Multiobjective optimization of SMB and varicol process for chiral separation. AIChE Journal, 48(12):2800-2816, 2002.

[74] Z. Zhong and F. You. Oil spill response planning with consideration of physicochemical evolution of the oil slick: A multiobjective optimization approach. Computers and Chemical Engineering, 35(8):1614-1630, 2011.

[75] A. Zilinskas, E. S. Fraga, and A. Mackute. Data analysis and visualisation for robust multicriteria process optimisation. Computers and Chemical Engineering, 30(6-7):1061-1071, 2006. 